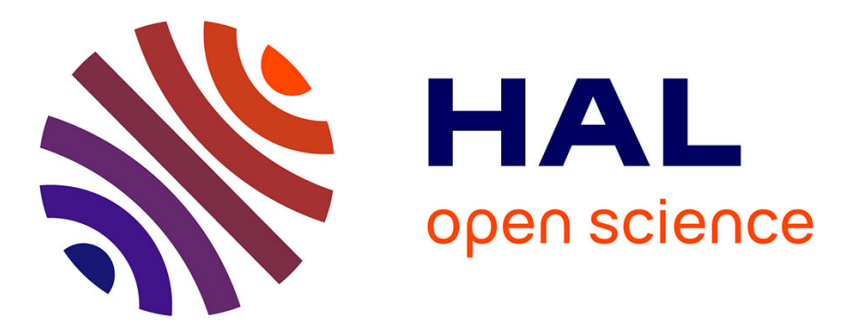

\title{
Relative chronology of crust formation on asteroid Vesta: Insights from the geochemistry of diogenites
} Jean-Alix J-A Barrat, A. Yamaguchi, B. Zanda, Claire Bollinger, Marcel Bohn

\section{To cite this version:}

Jean-Alix J-A Barrat, A. Yamaguchi, B. Zanda, Claire Bollinger, Marcel Bohn. Relative chronology of crust formation on asteroid Vesta: Insights from the geochemistry of diogenites. Geochimica et Cosmochimica Acta, 2010, 74 (21), pp.6218-6231. 10.1016/j.gca.2010.07.028 . insu-00517866

\section{HAL Id: insu-00517866 \\ https://hal-insu.archives-ouvertes.fr/insu-00517866}

Submitted on 25 Feb 2013

HAL is a multi-disciplinary open access archive for the deposit and dissemination of scientific research documents, whether they are published or not. The documents may come from teaching and research institutions in France or abroad, or from public or private research centers.
L'archive ouverte pluridisciplinaire HAL, est destinée au dépôt et à la diffusion de documents scientifiques de niveau recherche, publiés ou non, émanant des établissements d'enseignement et de recherche français ou étrangers, des laboratoires publics ou privés. 


\title{
Relative chronology of crust formation on asteroid Vesta: Insights from the geochemistry of diogenites
}

\author{
Jean-Alix Barrat ${ }^{\mathrm{a}, \mathrm{b}, *}$, Akira Yamaguchi ${ }^{\mathrm{c}}$, Brigitte Zanda ${ }^{\mathrm{d}}$, \\ Claire Bollinger $^{\mathrm{a}, \mathrm{b}}$, Marcel Bohn ${ }^{\mathrm{a}, \mathrm{b}}$ \\ ${ }^{a}$ Université Européenne de Bretagne, France \\ ${ }^{\mathrm{b}}$ Université de Brest, CNRS UMR 6538 (Domaines Océaniques), I.U.E.M., Place Nicolas Copernic, 29280 Plouzané Cedex, France \\ ${ }^{\mathrm{c}}$ Antarctic Meteorite Research Center, National Institute of Polar Research, 10-3 Midoricho, Tachikawa, Tokyo 190-8518, Japan \\ ${ }^{\mathrm{d}}$ Laboratoire de Minéralogie et de Cosmochimie du Muséum, CNRS UMR 7202, Muséum National d'Histoire Naturelle, \\ CP52, 57 rue Cuvier, 75005 Paris, France
}

Received 5 April 2010; accepted in revised form 26 July 2010; available online 12 August 2010

\begin{abstract}
The eucrites and diogenites are meteorites that probably originate from asteroid 4-Vesta. The upper part of the crust of this body is certainly composed of eucrites which are basaltic or gabbroic rocks. Diogenites are ultramafic cumulates whose relationships with eucritic lithologies are unknown. Here, we show that the orthopyroxenes of some diogenites display very deep negative $\mathrm{Eu}$ anomalies ( $\mathrm{Eu} / \mathrm{Eu}$ * close to 0.1 or lower). The contamination of the parental magmas of diogenites by melts derived by partial melting of the eucritic crust can satisfactorily explain the range of the Eu anomalies displayed by diogenites. Thus, these anomalies are the first firm indication that parental melts of diogenites have intruded the eucritic crust, and consequently are younger than eucrites.
\end{abstract}

(C) 2010 Elsevier Ltd. All rights reserved.

\section{INTRODUCTION}

Achondrites preserve a record of the magmatic processes that occurred during the differentiation of small planetary bodies. The howardite-eucrite-diogenite (HED) meteorites provide us with the largest sampling of magmatic rocks from such a body, probably asteroid 4-Vesta (Drake, 2001; McSween et al., 2010), whose surface will be studied next year by the Dawn spacecraft (Russell et al., 2007). Available lithologies comprise both a variety of basaltic or gabbroic rocks (eucrites) and ultramafic cumulates (diogenites). With more than 200 meteorites (including pairings), diogenites constitute one of the largest groups of achondrites. Almost all these rocks are orthopyroxenites, chiefly composed of magnesian orthopyroxene crystals, with minor

\footnotetext{
* Corresponding author at: Université de Brest, CNRS UMR 6538 (Domaines Océaniques), I.U.E.M., Place Nicolas Copernic, 29280 Plouzané Cedex, France. Tel.: +33 (0) 298498711.

E-mail address: barrat@univ-brest.fr(J.-A. Barrat).
}

chromite, silica, troilite, metal, and occasionally olivine, diopside, plagioclase, and rare phosphate (Mittlefehldt, 1994). A few diogenites display high modal abundances of olivine (harzburgites) and were seen as possible mantle lithologies (Sack et al., 1991). It is now widely accepted that all these rocks, including the "harzburgitic diogenites" (Beck and McSween, 2010), are cumulates but the exact compositions of their parental melts are still not well constrained (Mittlefehldt, 1994, 2000; Fowler et al., 1995; Barrat, 2004; Barrat et al., 2008; Shearer et al., 2010). We investigated the trace element geochemistry of diogenites because these lithologies provide an important record of the magmatic history of their parent body. They present the biggest challenge to existing models for the magma genesis and the structure of Vesta, which are at present largely based on the data obtained on eucritic lithologies. Currently there is little agreement about the genetic links and the relative chronology between eucrites and diogenites. It has been proposed that they formed during the cooling of a global magma ocean (Righter and Drake, 1997; Ruzicka et al., 1997; Warren, 1997). Alternatively, these rocks are 
not necessarily cogenetic (Mittlefehldt, 1994; Fowler et al., 1995). The parental melts of diogenites could have been produced by remelting of ultramafic cumulates previously crystallized in the magma ocean (Barrat, 2004; Barrat et al., 2008).

Here, we report new trace element abundances for a large set of diogenites, which show that the parental magmas of many of them have been contaminated by liquids produced by remelting of the eucritic crust. This demonstrates that diogenites formed after eucrites, and should be emplaced within the eucritic crust of Vesta rather than forming a lower layer as commonly thought.

\section{SAMPLES AND METHODS}

We analyzed samples from 33 different diogenites during the course of the study: Bilanga, Johnstown, Roda, Shalka, Tatahouine (fall), Dhofar (Dho) 700, Northwest Africa (NWA) 1461, NWA 1877, NWA 2629, NWA 3143, NWA 4223, NWA 4272, NWA 4664, NWA 5480, NWA 5613, Allan Hills (ALHA) 77256, Asuka (A-) 87147, A-880785, A-880946, A-881377, A-881526, A-881548, A-881838, A-881839, A-881944, Grosvenor Mountains (GRO) 95555, Meteorite Hills (MET) 00422, MET 00424, MET 00425, MET 00436, Miller Range (MIL) 07001, MIL 07003, Yamato (Y-) 74013 and Y-74097. The source of the samples and their main petrographical features are summarized in Table 1.

The major-element compositions of the orthopyroxenes were determined by electron microprobe analysis using a JEOL JXA8200 at National Institute of Polar Research, Tokyo, or a Cameca SX100 at Ifremer, Plouzané. All analyses used wavelength dispersive spectrometers at $15 \mathrm{kV}$ accelerating voltage, $10-30 \mathrm{nA}$ beam current. Minerals and metal standards were used for calibration.

Powders, prepared using a boron-carbide mortar and pestle, were leached in $6 \mathrm{M} \mathrm{HCl}\left(130{ }^{\circ} \mathrm{C}, 30-40 \mathrm{~min}\right)$. Residues (rinsed five times in ultrapure water) and unleached powders were completely dissolved using the procedure described by Barrat et al. (2008). Elemental abundances were determined using a high-resolution inductively coupled plasma-mass spectrometer Thermo Element 2 at Institut Universitaire Européen de la Mer (IUEM), Plouzané. Results on international standards have been repeatedly reported previously (Barrat et al., 2007, 2008; Barrat and Bollinger, 2010). Based on standards and many sample duplicates, the $1 \sigma$ analytical uncertainties for abundances and trace element ratios (e.g., Eu/Eu*, where $\mathrm{Eu}^{*}$ is the expected Eu concentration for a smooth CI-normalized REE pattern, such that $\mathrm{Eu}_{n}^{*}=\left(\mathrm{Sm}_{n} \times \mathrm{Gd}_{n}\right)^{1 / 2}$ ) are in most cases much better than $3 \%$. The results are given in Table 2, along with data obtained previously on some unleached powders (Bilanga, A-881526, A-881548), and three additional residues (Dho 700, NWA 4215 and A-881839,22) which were obtained using comparable procedures (Barrat et al., 2006, 2008).

One of the samples (MET 00424) displays light REE abundances which are too low to be accurately estimated using this procedure. Consequently we have separated the REEs from approximately $100 \mathrm{mg}$ of sample and concentrated the REEs in approximately $3 \mathrm{ml}$, using a simple ion-exchange chromatography procedure (Barrat et al., 1996). The accuracies are estimated to be better than $20 \%$ for $\mathrm{La}, \mathrm{Ce}$ and $\mathrm{Pr}$, better than $10 \%$ for $\mathrm{Sm}$ and $\mathrm{Nd}$, and better than 5\% for the other REEs, except Eu whose concentrations are indicative.

Finally, we have tried to determine the trace element abundances of the sparse plagioclase grains in Dhofar 700 by laser-ICP-MS (Barrat et al., 2009). Most of the crystals were too small or too thin to be correctly analyzed but we obtained a single analysis with acceptable statistics $(<10 \%)$, which is given in Table 2 .

\section{RESULTS AND DISCUSSION}

\subsection{Why leach the powders?}

Most of the available trace element data on diogenites to date have been previously obtained on bulk rock aliquots (Fukuoka et al., 1977; Mittlefehldt, 1994, 2000; Barrat et al., 2008). The interpretation of these analyses is difficult. At first glance, it can be assumed that the compositions of these fractions match satisfactorily those of the orthopyroxene crystals. Unfortunately, the trace element abundances are often hampered by minute amounts of minor phases such as metal and sulfides for siderophile or chalcophile elements, or phosphates for rare earth elements (REE) and Sr. Furthermore, a large number of diogenites are finds from hot deserts or Antarctica. These meteorites have suffered terrestrial weathering and contain secondary phases, whose contribution to the trace element budget can be significant (Barrat et al., 1999, 2006, 2008). These drawbacks could in principle be overcome by using, for example, ion-probe analyses of orthopyroxenes. Unfortunately, the abundances of many incompatible elements of interest are often very low (e.g., Eu generally much less than $10 \mathrm{ng} / \mathrm{g}$ ) and difficult to determine with appropriate accuracies using in-situ analytical procedures (e.g., Fowler et al., 1995). However, phosphates, and most of the secondary phases (e.g., rust, carbonates, ...) are easily removed from a diogenite powder using, for example, hot hydrochloric acid, leaving a residue essentially made of orthopyroxene. Thus, some trace element abundances or ratios (e.g., Sr, REE, Eu/Eu*) of orthopyroxenes can be deduced from those of the residues after leaching (e.g., Barrat et al., 2008). As an example, we have analyzed a large orthopyroxene clast prepared from the Johnstown diogenite. Johnstown has been extensively analyzed previously (Floran et al., 1981; Mittlefehldt, 1994; Barrat et al., 2008), and the REE abundances obtained on numerous bulk fractions are extremely variable. Some of the fractions display a marked light REE enrichment which has been previously ascribed to the involvement of a light REE component, possibly a trapped melt (Floran et al., 1981) or more likely a phosphate phase. A fraction of the powder (about $140 \mathrm{mg}$ ) was successively leached in $2 \mathrm{M} \mathrm{HCl}\left(50{ }^{\circ} \mathrm{C}, 30 \mathrm{~min}\right)$, and subsequently in $6 \mathrm{~N} \mathrm{HCl}\left(130{ }^{\circ} \mathrm{C}, 30 \mathrm{~min}\right)$. The first $\mathrm{HCl}$ fraction contained detectable amounts of $\mathrm{P}$, which indicated that a phosphate phase has been leached. Its trace element abundances (Table 2) have been calculated from the composition of the $\mathrm{HCl}$ fraction assuming a $\mathrm{P}_{2} \mathrm{O}_{5}$ concentration fixed to 
Table 1

Details of meteorites studied ( $\mathrm{D}$, diogenite; Ol D, olivine diogenite). $\mathrm{FeO} / \mathrm{MgO}$ ( $\mathrm{wt}^{\%} \% / \mathrm{wt} \%$ ) ratios from this study except $\mathrm{M}$ (Mittlefehldt, 1994), D (Domanik et al., 2004), B (Barrat et al., 2008), and S (Shearer et al., 2010).

\begin{tabular}{|c|c|c|c|c|c|}
\hline & Source & Type & $\mathrm{FeO} / \mathrm{MgO}$ & & Comment \\
\hline Falls & & & Bulk rock & Orthopyroxene & \\
\hline Tatahouine & MNHN & $\mathrm{D}$ & & 0.53 & Large opx crystal-two subsamples \\
\hline Johnstown & AMNH & $\mathrm{D}$ & & $0.57^{\mathrm{M}}$ & Large opx clast \\
\hline Shalka & J.A. Barrat & $\mathrm{D}$ & & $0.59^{\mathrm{M}}$ & Opx fraction \\
\hline Bilanga & A. Carion/J.A. Barrat & $\mathrm{D}$ & & $0.45^{\mathrm{D}}$ & Same powder as Barrat (2004) and a large opx crystal \\
\hline Roda & MNHN & Ol D & & $0.61^{\mathrm{M}}$ & Two samples (bulk and opx fraction) \\
\hline \multicolumn{6}{|l|}{ Hot-desert finds } \\
\hline Dhofar 700 & J.A. Barrat & $\mathrm{D}$ & & 0.84 & Unbrecciated, unequilibrated \\
\hline NWA 1461 & ROM & $\mathrm{D}$ & & 0.30 & \\
\hline NWA 1877 & J.A. Barrat & $\mathrm{D}$ & & 0.55 & \\
\hline NWA 2629 & J.A. Barrat & $\mathrm{D}$ & & & Paired with NWA 1877 \\
\hline NWA 3143 & J.A. Barrat & $\mathrm{D}$ & & 0.64 & \\
\hline NWA 4215 & ENSL & $\mathrm{D}$ & $0.67^{\mathrm{B}}$ & & Unbrecciated, unequilibrated, data from Barrat et al. (2006) \\
\hline NWA 4223 & J.A. Barrat & Ol D & & 0.62 & Unbrecciated, opx rich fragment \\
\hline NWA 4272 & M. Franco & $\mathrm{D}$ & $0.54^{\mathrm{B}}$ & & Same powder as Barrat et al. (2008) \\
\hline NWA 4664 & J.A. Barrat & $\mathrm{D}$ & & & \\
\hline NWA 5480 & J.A. Barrat & Ol D & & 0.59 & Unbrecciated, opx rich fragment \\
\hline NWA 5613 & UBO & $\mathrm{D}$ & & 0.71 & \\
\hline \multicolumn{6}{|l|}{ Antarctic finds } \\
\hline ALHA 77256,65 & MWG & Ol D & & 0.58 & \\
\hline A- 87147,21 & NIPR & $\mathrm{D}$ & & 0.59 & \\
\hline A- 880785,56 & NIPR & $\mathrm{D}$ & & 0.62 & \\
\hline A- 880936,22 & NIPR & $\mathrm{D}$ & & 0.61 & \\
\hline A-881377,62 & NIPR & $\mathrm{D}$ & & 0.59 & \\
\hline A- 881526,56 & NIPR & $\mathrm{D}$ & $0.59^{\mathrm{B}}$ & & Same powder as Barrat et al. (2008) \\
\hline A- 881548,23 & NIPR & $\mathrm{Ol} \mathrm{D}$ & $0.60^{\mathrm{B}}$ & & Same powder as Barrat et al. (2008) \\
\hline A- 881838,31 & NIPR & $\mathrm{D}$ & & 0.75 & \\
\hline A- 881839,22 & NIPR & $\mathrm{D}$ & $0.79^{\mathrm{B}}$ & & Data from Barrat et al. (2008) \\
\hline A-881944,61 & NIPR & $\mathrm{D}$ & & & \\
\hline GRO 95555,39 & MWG & $\mathrm{D}$ & & 0.55 & Unbrecciated \\
\hline MET 00422,14 & MWG & $\mathrm{D}$ & $0.69^{\mathrm{B}}$ & & \\
\hline MET 00424,24 & MWG & $\mathrm{D}$ & & $0.74^{\mathrm{S}}$ & \\
\hline MET 00425,16 & MWG & $\mathrm{D}$ & $0.37^{\mathrm{B}}$ & & \\
\hline MET 00436,31 & MWG & $\mathrm{D}$ & $0.90^{\mathrm{B}}$ & & \\
\hline MIL 07001,10 & MWG & Ol D & & 0.55 & Unbrecciated \\
\hline MIL 07003,9 & MWG & $\mathrm{D}$ & & 0.98 & \\
\hline $\mathrm{Y}-74013,121$ & NIPR & $\mathrm{D}$ & & 0.65 & \\
\hline Y-74097,56 & NIPR & $\mathrm{D}$ & & 0.60 & \\
\hline
\end{tabular}

Abbreviations: AMNH, American Museum of Natural History, New York; MWG, NASA Meteorite Working Group; MNHN, Muséum National d'Histoire Naturelle, Paris; NIPR, National Institute of Polar Research, Tokyo; ROM, Royal Ontario Museum; UBO, Université de Bretagne Occidentale.

$42 \mathrm{wt} \%$. Unsurprisingly, the calculated phosphate is REErich (Table 2), and displays a marked light REE enrichment (Fig. 1). The residue is light REE depleted as expected for a pure orthopyroxene fraction (Fig. 1). The trace element content of the unleached powder is well accounted for by a mixture of $99.994 \mathrm{wt} \%$ residue and only $0.006 \mathrm{wt} \%$ phosphate. Hence, traces of phosphates explain the spread of light REE abundances of the unleached samples.

The involvement of phosphates has been checked in all the diogenites from Antarctica we have analyzed. The comparison of the REE ratios of the residues and the unleached powder demonstrate that such phases occur in many diogenites. The $\mathrm{P}$ contents of the residues were always negligible (much less than $5 \mu \mathrm{g} / \mathrm{g}$ in most cases) demonstrating the efficiency of our leaching procedure. The residues are gener- ally much more light REE depleted than the unleached powders (Fig. 2). In many cases, the residues display a negative $\mathrm{Eu}$ anomaly much more pronounced than the unleached powder and such an effect is well explained by the traces of phosphate in the samples. Notice that the occurrence of phosphate has virtually no effect on the heavy REE abundances or ratios (Table 2 and Fig. 2).

Diogenites found in hot deserts often contain secondary minerals such as carbonates, rust and sometimes minor amounts of sulfates. Despite the leaching step, many residues obtained from hot-desert diogenites still display high $\mathrm{Ba}$ and $\mathrm{Sr}$ abundances. We ascribe these high concentrations to sulfates (barite, gypsum), which were not dissolved. The impact of these phases on the REEs is uncertain. As we will see below, the REE abundances of the residues 
Table 2

Trace element abundances (in $\mathrm{ng} / \mathrm{g}$, except $*$, in $\mu \mathrm{g} / \mathrm{g}$ ) in unleached fractions $(\mathrm{U})$ and residues after leaching $(\mathrm{R})$ of diogenites.

\begin{tabular}{|c|c|c|c|c|c|c|c|c|c|c|c|c|c|c|c|c|c|c|c|}
\hline & $\mathrm{Sr}$ & $\mathrm{Y}$ & $\mathrm{Zr}$ & $\mathrm{Ba}$ & $\mathrm{La}$ & $\mathrm{Ce}$ & $\operatorname{Pr}$ & $\mathrm{Nd}$ & $\mathrm{Sm}$ & $\mathrm{Eu}$ & Gd & $\mathrm{Tb}$ & Dy & Ho & Er & $\mathrm{Yb}$ & $\mathrm{Lu}$ & $\mathrm{Hf}$ & $\mathrm{Eu} / \mathrm{Eu}^{*}$ \\
\hline \multicolumn{20}{|l|}{ Falls } \\
\hline Tatahouine-R1 & 22 & 209 & 112 & 4 & 1.52 & 3.92 & 0.61 & 3.64 & 2.16 & 0.28 & 8.26 & 2.37 & 25.2 & 7.99 & 33.8 & 64.3 & 12.4 & 5.5 & 0.20 \\
\hline Tatahouine-R2 & 17 & 236 & 91 & - & 1.17 & 3.24 & 0.54 & 3.24 & 2.14 & 0.25 & 7.32 & 2.28 & 24.4 & 7.91 & 34 & 62.9 & 12.4 & 4.5 & 0.20 \\
\hline Johnstown-opx-U & 1002 & 1774 & 3617 & 4260 & 142 & 386 & 58.7 & 299 & 107 & 9.62 & 170 & 34.2 & 255 & 62 & 197 & 233 & 38.4 & 88 & 0.22 \\
\hline Johnstown-opx-R & 166 & 1676 & 3293 & 1732 & 22.4 & 129 & 30.8 & 203 & 89 & 3.37 & 154 & 31.4 & 239 & 58.7 & 189 & 227 & 37.3 & 82.7 & 0.09 \\
\hline Johnstown-phosph. & $6929^{*}$ & $1658^{*}$ & $698^{*}$ & $3104 *$ & $2199 *$ & $4839^{*}$ & $546^{*}$ & $1969^{*}$ & $325^{*}$ & $37.7^{*}$ & $324^{*}$ & $47.7^{*}$ & $263^{*}$ & $53.1^{*}$ & $134^{*}$ & $113^{*}$ & $15.2^{*}$ & $11.4^{*}$ & 0.36 \\
\hline Shalka-R & 143 & 154 & 214 & 33 & 7.65 & 20.3 & 3.12 & 16.5 & 5.85 & 1.16 & 8.95 & 2.01 & 18.1 & 5.16 & 19.69 & 33.8 & 6.45 & 3.9 & 0.49 \\
\hline Bilanga-U & 951 & 994 & 715 & 210 & 11.3 & 48.7 & 10.1 & 69.3 & 42.6 & 4.15 & 74.6 & 17.8 & 145 & 37.1 & 119 & 129 & 21.1 & 24.7 & 0.23 \\
\hline Bilanga-R1 & 110 & 929 & 537 & 61 & 4.54 & 23.1 & 5.66 & 43.2 & 29.6 & 1.76 & 67.8 & 15.8 & 126 & 31.9 & 107 & 127 & 20.3 & 20.9 & 0.12 \\
\hline Bilanga-R2 & 92 & 890 & 555 & 53 & 3.85 & 20.8 & 4.98 & 40.2 & 28 & 1.56 & 61.8 & 14.8 & 122 & 30.8 & 103 & 123 & 20.1 & 21.3 & 0.11 \\
\hline Bilanga-opx-R & 130 & 948 & 800 & 75 & 7.18 & 29.5 & 6.58 & 47.1 & 31.8 & 2.37 & 67.2 & 15.7 & 125 & 31.6 & 104 & 123 & 20 & 25.3 & 0.16 \\
\hline Roda-opx-R & 147 & 2155 & 4931 & 322 & 20.2 & 133 & 35.4 & 239 & 115 & 4.07 & 193 & 41.1 & 305 & 74.9 & 239 & 282 & 46 & 128.9 & 0.08 \\
\hline Roda-R & 539 & 1954 & 3219 & 357 & 36 & 148 & 32.8 & 212 & 99.3 & 6.7 & 173 & 36.1 & 273 & 66.2 & 218 & 261 & 42.4 & 95.8 & 0.16 \\
\hline \multicolumn{20}{|l|}{ Hot-desert finds } \\
\hline Dho 700A-R1 & 170 & 907 & 283 & 85 & 2.75 & 12.7 & 2.34 & 18.9 & 17.1 & 0.84 & 47.1 & 12.9 & 116 & 32.1 & 108 & 126 & 19.84 & 13.9 & 0.09 \\
\hline Dho 700A-R2 & 360 & 910 & 330 & 120 & 4.7 & 25 & 3.2 & 21.5 & 17.5 & 1.1 & 47.2 & 13.1 & 116 & 31.3 & 107 & 127 & 20.2 & 15 & 0.12 \\
\hline Dho 700B-R1 & 5150 & 920 & 300 & 1080 & 77.2 & 380 & 23.5 & 23.9 & 18 & 1.1 & 48 & 13.7 & 119 & 32.5 & 111 & 128 & 19.7 & 16 & 0.11 \\
\hline Dho 700B-R2 & 2010 & 920 & 290 & 450 & 16 & 161.3 & 9.8 & 22.4 & 18.5 & 0.9 & 47.2 & 13.8 & 122 & 32.4 & 111 & 127 & 19.9 & 16 & 0.09 \\
\hline Dho 700C-R & 148 & 784 & 803 & 193 & 7.78 & 21.6 & 3.54 & 21.1 & 14.2 & 0.99 & 35.9 & 10.1 & 94.8 & 26.1 & 92.2 & 116 & 18.94 & 13.9 & 0.13 \\
\hline Dho 700C-plagio. & $101^{*}$ & 381 & & $11.6^{*}$ & 449 & 841 & 112 & 398 & 85 & 418 & 94 & & 78 & & & 18 & & & 14.3 \\
\hline NWA $1461-R$ & 1031 & 300 & 907 & 567 & 68.2 & 181 & 27.1 & 134 & 39.4 & 10.6 & 46.2 & 7.91 & 50.2 & 10.6 & 30.8 & 32.9 & 5.03 & 24.5 & 0.76 \\
\hline NWA 1877A-R & 310 & 193 & 538 & 347 & 33.9 & 70.9 & 9.38 & 45 & 14.5 & 2.13 & 20.3 & 3.72 & 27.5 & 6.23 & 21.2 & 34.7 & 6.8 & 14.8 & 0.38 \\
\hline NWA 1877B-R & 388 & 196 & 925 & 872 & 70.5 & 134 & 15.9 & 63.5 & 15.4 & 2.98 & 20 & 3.38 & 24.6 & 6.21 & 21.5 & 33.2 & 6.5 & 16.9 & 0.52 \\
\hline NWA 1877C-R & 670 & 315 & 1557 & 2142 & 98.2 & 186 & 22 & 88.2 & 21.6 & 4.67 & 30.7 & 5.45 & 40.6 & 10 & 34.1 & 49.5 & 9.3 & 32.3 & 0.55 \\
\hline NWA 2629-R & 328 & 237 & 784 & 394 & 42.7 & 86.6 & 11.8 & 54.8 & 18 & 2.94 & 23.7 & 4.37 & 32.9 & 7.74 & 26.3 & 39.1 & 7.33 & 14.2 & 0.44 \\
\hline NWA 3143-R & 286 & 3347 & 5040 & 372 & 67.2 & 288 & 60.3 & 364 & 164 & 5.4 & 311 & 62.6 & 469 & 110 & 343 & 372 & 58 & 138 & 0.07 \\
\hline NWA 4215-R & 1243 & 819 & 221 & 3190 & 10.4 & 26.4 & 3.8 & 21.1 & 13.4 & 3.12 & 35.7 & 9.85 & 99.7 & 28.4 & 106 & 147 & 26.2 & 10.5 & 0.44 \\
\hline NWA 4223-R & 579 & 1296 & 1637 & 12487 & 21.9 & 70.3 & 13.2 & 86.5 & 47.4 & 5.59 & 93.2 & 21.5 & 172 & 43.3 & 144 & 178 & 29.7 & 51.2 & 0.26 \\
\hline NWA 4272-U & 2362 & 2731 & 3552 & 1753 & 131 & 423 & 71.1 & 395 & 158 & 17.8 & 245 & 52.7 & 403 & 98.9 & 307 & 326 & 52.5 & 108 & 0.28 \\
\hline NWA 4272-R & 400 & 2623 & 4384 & 319 & 82.5 & 306 & 59.6 & 360 & 155 & 4.44 & 258 & 52.0 & 381 & 91.0 & 287 & 322 & 52.8 & 126 & 0.07 \\
\hline NWA 4664-R1 & 3703 & 2756 & 7077 & 15093 & 125 & 381 & 70.4 & 422 & 184 & 25.9 & 302 & 59.9 & 418 & 96.6 & 298 & 313 & 48.1 & 198 & 0.34 \\
\hline NWA 4664-R2 & 3033 & 2027 & 4474 & 21146 & 78.3 & 275 & 51.7 & 313 & 133 & 15.9 & 204 & 40.4 & 296 & 69.6 & 212 & 225 & 34.3 & 114 & 0.3 \\
\hline NWA $5480-R$ & 36 & 224 & 283 & 84 & 2.82 & 6.24 & 0.73 & 2.8 & 1.56 & 0.19 & 4.96 & 1.79 & 21.3 & 7.09 & 30.8 & 55.4 & 10.6 & 2.3 & 0.21 \\
\hline NWA 5613-R & 190 & 3297 & 8275 & 576 & 104 & 353 & 70.8 & 444 & 202 & 3.41 & 329 & 67.4 & 499 & 117 & 371 & 408 & 65.4 & 226 & 0.04 \\
\hline \multicolumn{20}{|l|}{ Antarctican finds } \\
\hline ALHA $77256,65-\mathrm{U}$ & 297 & 1744 & 2223 & 127 & 95.6 & 276 & 42.2 & 216 & 81.8 & 5.88 & 140 & 30.2 & 238 & 60.5 & 195 & 229 & 37.7 & 67.5 & 0.17 \\
\hline ALHA $77256,65-\mathrm{R}$ & 256 & 1694 & 1961 & 65 & 76.7 & 237 & 37.7 & 198 & 75.8 & 5.67 & 132 & 29.1 & 231 & 58.6 & 193 & 227 & 37.4 & 61.9 & 0.17 \\
\hline A-87147,21-U & 813 & 1527 & 1853 & 565 & 64.1 & 165 & 28.3 & 164 & 68.7 & 9.98 & 125 & 26.9 & 211 & 52.6 & 175 & 219 & 35.3 & 59.0 & 0.33 \\
\hline A-87147,21-R & 736 & 1496 & 1907 & 604 & 52.2 & 158 & 30.2 & 176 & 70.1 & 9.81 & 123 & 26.8 & 205 & 52.2 & 173 & 213 & 34.6 & 60.3 & 0.32 \\
\hline
\end{tabular}


Table 2 (continued)

\begin{tabular}{|c|c|c|c|c|c|c|c|c|c|c|c|c|c|c|c|c|c|c|c|}
\hline & $\mathrm{Sr}$ & $\mathrm{Y}$ & $\mathrm{Zr}$ & $\mathrm{Ba}$ & $\mathrm{La}$ & $\mathrm{Ce}$ & $\operatorname{Pr}$ & $\mathrm{Nd}$ & $\mathrm{Sm}$ & $\mathrm{Eu}$ & Gd & $\mathrm{Tb}$ & Dy & Ho & $\mathrm{Er}$ & $\mathrm{Yb}$ & $\mathrm{Lu}$ & $\mathrm{Hf}$ & $\mathrm{Eu} / \mathrm{Eu}^{*}$ \\
\hline A-880785,56-U & 644 & 468 & 324 & 427 & 14.4 & 33.0 & 4.76 & 25.9 & 12.3 & 2.11 & 25.5 & 6.54 & 57.0 & 15.9 & 56.3 & 83.7 & 14.4 & 11.8 & 0.36 \\
\hline A- $880785,56-R$ & 145 & 463 & 293 & 69 & 3.87 & 13.5 & 2.84 & 18.3 & 10.6 & 1.89 & 23.9 & 6.19 & 56.3 & 15.7 & 57.6 & 85.5 & 14.6 & 10.9 & 0.36 \\
\hline A-880936,22-U & 295 & 389 & 247 & 138 & 8.16 & 22.0 & 3.35 & 18.5 & 9.44 & 1.88 & 21.3 & 5.34 & 47.8 & 13.2 & 48.1 & 71.9 & 12.7 & 8.9 & 0.4 \\
\hline A-880936,22-R & 136 & 395 & 245 & 46 & 3.2 & 12.5 & 2.32 & 15.9 & 9.43 & 1.75 & 21.8 & 5.48 & 48.3 & 13.4 & 48.9 & 72.9 & 13.2 & 9.4 & 0.37 \\
\hline A-881377,62-U & 3256 & 1420 & 2083 & 1122 & 101 & 254 & 39.3 & 202 & 75.5 & 23.2 & 122 & 25.4 & 197 & 49.3 & 165 & 210 & 35.9 & 65.6 & 0.74 \\
\hline A-881377,62-R & 332 & 1390 & 2224 & 462 & 33.9 & 141 & 27.5 & 167 & 69.9 & 8.72 & 119 & 25.0 & 191 & 47.9 & 158 & 201 & 33.7 & 70.1 & 0.29 \\
\hline A-881526,56-U & 514 & 1329 & 1069 & 22 & 27.9 & 81.9 & 13.8 & 88.0 & 50.0 & 8.40 & 93.2 & 22.4 & 184 & 48.5 & 162 & 212 & 35.6 & 40.1 & 0.38 \\
\hline A-881526,56-R & 461 & 1420 & 1078 & 13 & 27.2 & 76.7 & 14.1 & 88.6 & 49.5 & 8.42 & 106 & 24.3 & 193 & 49.6 & 166 & 209 & 35.0 & 37.9 & 0.35 \\
\hline A-881548,23-U & 441 & 1681 & 1793 & 374 & 60.5 & 277 & 57.3 & 347 & 131 & 7.75 & 171 & 34.6 & 253 & 61.2 & 191 & 217 & 35.3 & 44.2 & 0.16 \\
\hline A-881548,23-R & 384 & 1897 & 1877 & 141 & 49.2 & 257 & 57.8 & 352 & 135 & 8.27 & 195 & 38.8 & 276 & 64.5 & 203 & 228 & 36.0 & 45.0 & 0.16 \\
\hline A-881838,31-U & 4703 & 3100 & 7796 & 3161 & 488 & 1297 & 185 & 905 & 274 & 48.1 & 372 & 67.9 & 473 & 107 & 326 & 348 & 54.8 & 209 & 0.46 \\
\hline A- $881838,31-R$ & 274 & 2589 & 6871 & 512 & 77.8 & 302 & 63.9 & 396 & 171 & 5.13 & 262 & 52.4 & 385 & 91.5 & 288 & 320 & 50.7 & 194 & 0.07 \\
\hline A-881839,22-U & 4676 & 2981 & 6961 & 2256 & 368 & 977 & 151 & 793 & 265 & 43.5 & 348 & 65.5 & 459 & 108 & 330 & 347 & 55.1 & 192 & 0.44 \\
\hline A-881839,22-R & 646 & 2564 & 6898 & 650 & 86.1 & 303 & 60.6 & 405 & 179 & 8.83 & 269 & 55.3 & 404 & 97.9 & 313 & 337 & 54.6 & 198 & 0.12 \\
\hline A-881944,61-U & 2348 & 2341 & 3842 & 1462 & 143 & 397 & 69.0 & 376 & 142 & 21.4 & 221 & 45.0 & 335 & 81.5 & 258 & 304 & 48.3 & 107 & 0.37 \\
\hline A-881944,61-R & 193 & 2150 & 3635 & 349 & 70.7 & 262 & 50.9 & 299 & 124 & 4.24 & 200 & 41.5 & 309 & 74.9 & 243 & 285 & 45.4 & 108 & 0.08 \\
\hline GRO 95555,39-U & 341 & 514 & 431 & 67 & 16.1 & 54.8 & 8.79 & 50.8 & 20.1 & 4.56 & 35.9 & 8.24 & 65.7 & 17.6 & 62.5 & 88.9 & 15.0 & 12.9 & 0.52 \\
\hline GRO 95555,39-R & 248 & 492 & 359 & 8 & 10.5 & 39.5 & 6.88 & 40.0 & 17.8 & 3.66 & 31.9 & 7.52 & 61.7 & 16.7 & 60.3 & 85.8 & 15.0 & 11.1 & 0.47 \\
\hline MET $00422,14-U$ & 115 & 224 & 95 & 69 & 5.67 & 13.9 & 2.00 & 10.6 & 5.01 & 1.04 & 9.46 & 2.93 & 26.5 & 7.39 & 28.0 & 42.0 & 7.80 & 3.5 & 0.46 \\
\hline MET $00422,14-\mathrm{R}$ & 82 & 210 & 84 & 37 & 4.28 & 11.6 & 1.71 & 8.9 & 4.20 & 1.10 & 9.94 & 2.63 & 24.2 & 7.03 & 26.1 & 40.8 & 7.30 & 3.1 & 0.52 \\
\hline MET $00424,24-U$ & 9.7 & 3.80 & 12 & 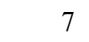 & 0.47 & 0.89 & 0.082 & 0.33 & 0.069 & 0.025 & 0.094 & 0.018 & 0.18 & 0.10 & 0.86 & 5.01 & 1.39 & 0.3 & 0.95 \\
\hline MET 00424,24-R & 3.3 & 2.97 & 19 & $\gamma$ & 0.20 & 0.42 & 0.040 & 0.17 & 0.036 & $<0.02$ & 0.045 & 0.0093 & 0.14 & 0.10 & 0.88 & 4.95 & 1.36 & 0.5 & $<1.5$ \\
\hline MET $00425,16-U$ & 325 & 457 & 792 & 534 & 23.5 & 95 & 13.3 & 73.7 & 26.7 & 4.29 & 42.0 & 8.41 & 62.2 & 15.9 & 52.1 & 67.1 & 10.7 & 20.9 & 0.39 \\
\hline MET $00425,16-\mathrm{R}$ & 316 & 372 & 801 & 212 & 20.4 & 74.3 & 13.7 & 78.5 & 29.4 & 3.95 & 41.6 & 7.80 & 54.5 & 12.9 & 41.4 & 51.5 & 8.60 & 21.7 & 0.34 \\
\hline MET 00436,31-U & 297 & 165 & 358 & 96 & 10.6 & 31.8 & 5.58 & 34 & 13.2 & 2.49 & 20.3 & 3.80 & 26.0 & 5.8 & 16.8 & 20.6 & 3.60 & 10.1 & 0.47 \\
\hline MET $00436,31-R$ & 314 & 163 & 259 & 37 & 5.99 & 21.7 & 4.39 & 28.5 & 11.7 & 2.44 & 19.5 & 3.64 & 25.3 & 5.73 & 17.3 & 21.0 & 3.59 & 8.5 & 0.49 \\
\hline MIL 07001,10-U & 2379 & 840 & 1822 & 1069 & 60.6 & 177 & 27.1 & 141 & 51.4 & 16.9 & 86.6 & 16.7 & 121 & 29 & 92.9 & 114 & 18.8 & 47.0 & 0.78 \\
\hline MIL 07001,10-R & 1868 & 902 & 1918 & 774 & 34.2 & 129 & 23.6 & 134 & 52.6 & 17.0 & 89.8 & 18.0 & 131 & 31.7 & 102 & 125 & 20.9 & 50.6 & 0.76 \\
\hline MIL 07003,9-U & 11974 & 4860 & 6789 & 7301 & 281 & 758 & 125 & 708 & 283 & 104 & 484 & 96.7 & 714 & 171 & 541 & 595 & 93.7 & 202 & 0.86 \\
\hline MIL 07003,9-R & 887 & 4540 & 6062 & 857 & 126 & 425 & 82.6 & 510 & 231 & 10.2 & 399 & 85.4 & 666 & 162 & 517 & 593 & 93.2 & 193 & 0.10 \\
\hline Y-74013,121A-U & 286 & 359 & 290 & 73 & 8.92 & 24.3 & 3.97 & 23.3 & 10.6 & 2.98 & 19.7 & 4.77 & 41.5 & 11.8 & 44.6 & 72.5 & 13.2 & 9.1 & 0.63 \\
\hline $\mathrm{Y}-74013,121 \mathrm{~A}-\mathrm{R}$ & 313 & 380 & 309 & 144 & 9.89 & 26.1 & 4.67 & 25.6 & 11.1 & 3.23 & 21.2 & 5.06 & 44.2 & 12.7 & 48.0 & 75.6 & 13.9 & 9.9 & 0.65 \\
\hline 74013 & 77 & Y-315 & 201 & 11 & 4.80 & 15. & 2.6 & 15.2 & 7.61 & 1.39 & 16.3 & 3.85 & 36.7 & 10.8 & 40.9 & 66.4 & 12.5 & 7.4 & 0.38 \\
\hline Y-74097,56-U & 273 & 381 & 294 & 43 & 8.05 & 22.6 & 3.84 & 22.5 & 10.8 & 3.10 & 21.6 & 5.13 & 44.9 & 13.0 & 47.5 & 74.0 & 13.7 & 9.7 & 0.62 \\
\hline Y-74097,56-R & 132 & 397 & 325 & 19 & 7.14 & 21.4 & 3.83 & 22.9 & 11.1 & 2.27 & 22.8 & 5.39 & 47.7 & 13.5 & 49.2 & 76.8 & 13.9 & 10.7 & 0.44 \\
\hline Uncert. $(1 \sigma)$ & $<3 \%$ & $<2 \%$ & $<7 \%$ & $<2 \%$ & $<3 \%$ & $<3 \%$ & $<3 \%$ & $<3 \%$ & $<3 \%$ & $<3 \%$ & $<3 \%$ & $<3 \%$ & $<3 \%$ & $<3 \%$ & $3 \%$ & $<3 \%$ & $<3 \%$ & $<5 \%$ & $<3 \%$ \\
\hline
\end{tabular}




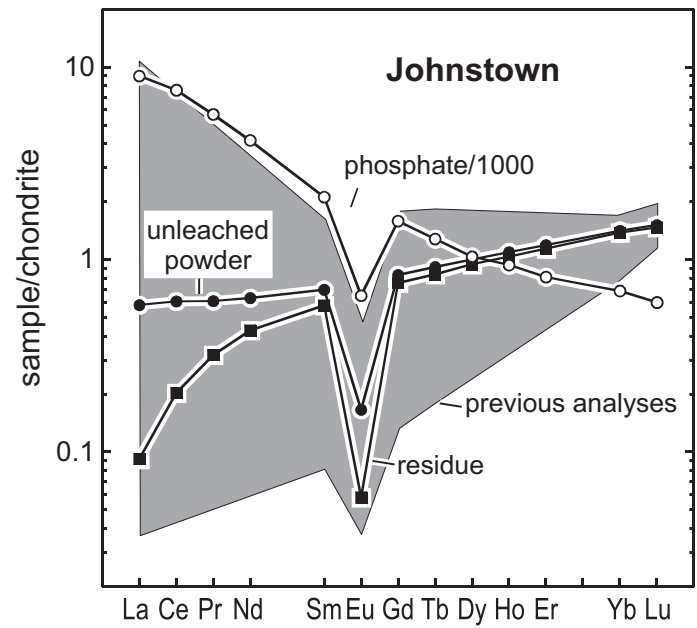

Fig. 1. REE patterns of the Johnstown diogenite (this work). The field of literature results is shown for comparison (Floran et al., 1981; Mittlefehldt, 1994). The reference chondrite is from Evensen et al. (1978).

obtained from hot desert and Antarctica diogenites are generally similar. However, a few samples (Dhofar 700, NWA 1461, NWA 1877/2629, NWA 5480) display spurious light REE enrichments (Figs. 3 and 4), which can be more likely seen as the fingerprint of the terrestrial weathering (Barrat et al., 1999, 2006, 2008).

Except for some weathered hot-desert samples, the REE abundances of the residues are certainly indistinguishable from those of the orthopyroxenes. A few percent of chromite or olivine in these fractions have no effect on the shape of the REE patterns because these phases display very low REE abundances. Traces of plagioclase are not removed by our procedure and could in principle explain a very limited part of the range of the $\mathrm{Eu} / \mathrm{Eu}^{*}$ values obtained here. This problem should not be overemphasized because plagioclase, when present, is only a minor phase. It can account only for a limited spread of the $\mathrm{Eu} / \mathrm{Eu}^{*}$ values in some cases (e.g., the Yamato-A diogenites), but certainly not for the striking negative Eu anomalies which are the key arguments of our study.

\subsection{Groupings}

Diogenites display a remarkable range of REE abundances (Fukuoka et al., 1977; Mittlefehldt, 1994, 2000; Barrat et al., 2008), and unsurprisingly, their orthopyroxenes (i.e., the residues) are no exceptions (Fowler et al., 1995). The $\mathrm{Yb}$ abundances obtained here range for example, from 5 to $593 \mathrm{ng} / \mathrm{g}$. More importantly, diogenitic orthopyroxenes are characterized by an impressive range of negative $\mathrm{Eu}$ anomalies $\left(\mathrm{Eu} / \mathrm{Eu}^{*}=0.04-0.76\right.$, Fig. 1), and heavy REE enrichments $\left((\mathrm{Dy} / \mathrm{Lu})_{n}=0.01-0.8\right)$. Neither the REE abundances, nor the REE ratios (e.g., Dy/Lu, $\mathrm{Eu} / \mathrm{Eu}^{*}$ ) are correlated with major elements (not shown). Before discussing the origin of the deep negative Eu anomalies and the range of the heavy REE enrichments shown by the diogenitic orthopyroxenes, the REE abundances allow us to group most of the samples analyzed here.

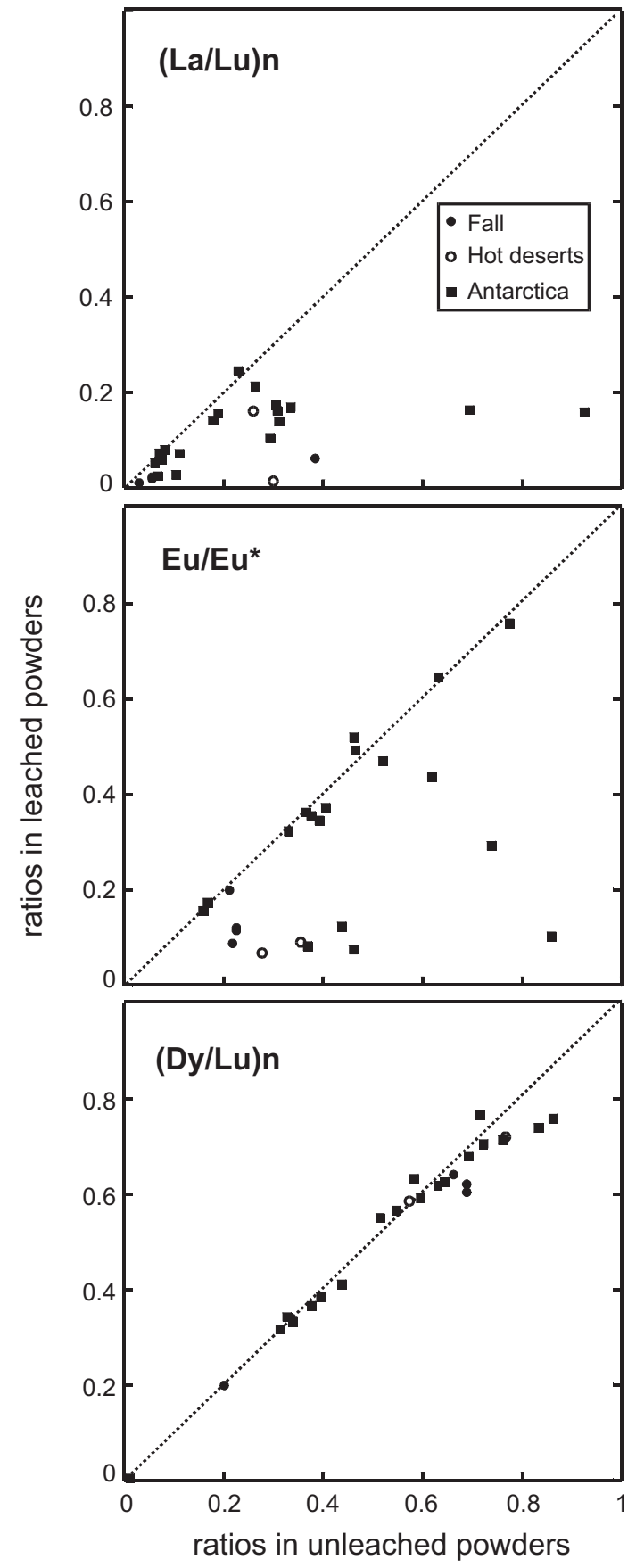

Fig. 2. Comparison of the $(\mathrm{La} / \mathrm{Lu})_{n}, \mathrm{Eu} / \mathrm{Eu}^{*}$, and $(\mathrm{Dy} / \mathrm{Lu})_{n}$ ratios in the residues and in the unleached powders.

\subsubsection{Tatahouine group}

Tatahouine and NWA 5480 display REE patterns with the same heavy REE enrichments and Eu anomalies. The similarities between the patterns of these two diogenites are so striking (Fig. 4), that it can be suggested that they certainly formed from similar parental melts, possibly from the same magmatic body.

\subsubsection{Yamato- $A$ group}

We have analyzed chips from Y-74013 and Y-74097, two paired diogenites found during the seventies in the 


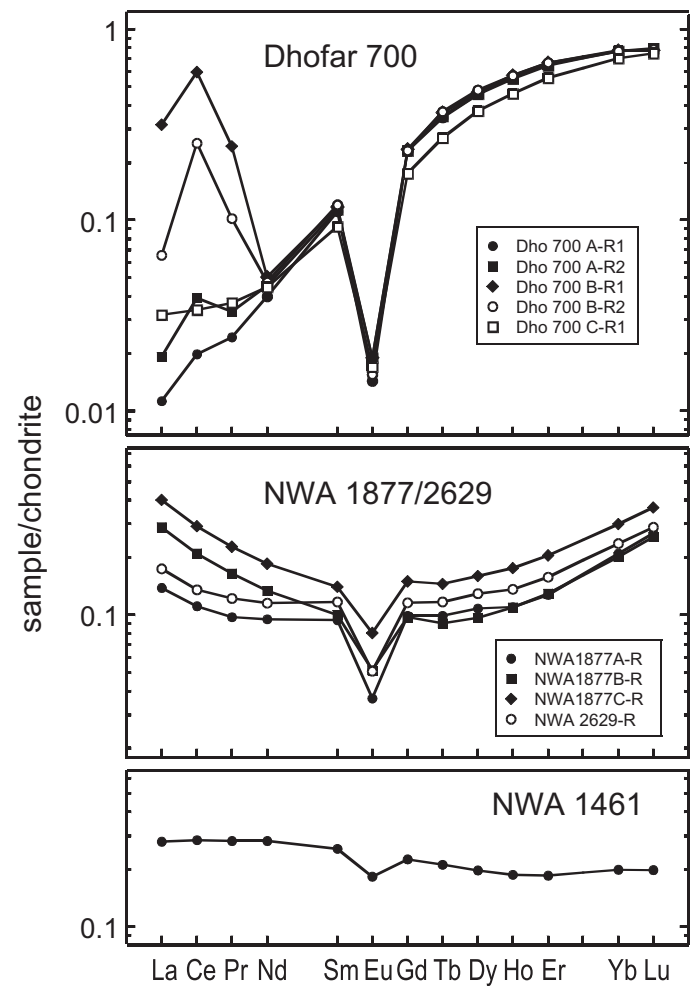

Fig. 3. REE patterns of the Dhofar 700, NWA 1877/2629 and NWA 1461 diogenites (residues after leaching). The reference chondrite is from Evensen et al. (1978). The $2 \sigma$ errors for the analyses are equivalent to the size of the data points.

Yamato meteorite field (Takeda et al., 1981). The shape of their REE patterns are similar (Fig. 4), except for the Eu anomalies $\left(\mathrm{Eu} / \mathrm{Eu}^{*}=0.38-0.65\right)$. These diogenites contain traces of plagioclase which can explain these small variations. Furthermore, three additional diogenites (A-880785, A-880936 and GRO 95555) display the same kind of REE patterns and could belong to the same group of samples.

\subsubsection{EETA 79002 group}

It has been previously shown that a series of diogenites from Antarctica (EETA 79002, A-881526, LAP 02216, LAP 03569, LAP 03630, MET 00422, MIL 03368) displays strikingly parallel REE patterns, and formed certainly from very similar melts (Barrat et al., 2008). Two of these diogenites have been reanalyzed here. The REE abundances in the unleached fractions and the residues are identical and confirm that these diogenites do not contain phosphate (Table 2). Moreover, NWA 4223, an unbrecciated olivine diogenite belongs to this group (Fig. 5).

\subsubsection{MET 00436 group}

MET 00425, MET 00436 and MIL 07001 orthopyroxenes display parallel REE patterns (Fig. 5). Notice that the $\mathrm{Eu}$ anomalies are different for the three residues $(\mathrm{Eu} /$ $\left.\mathrm{Eu}^{*}=0.34-0.76\right)$.

\subsubsection{Johnstown group}

The orthopyroxenes from Johnstown, Bilanga, Roda, A-881548 and Dhofar 700 (the least weathered fraction)

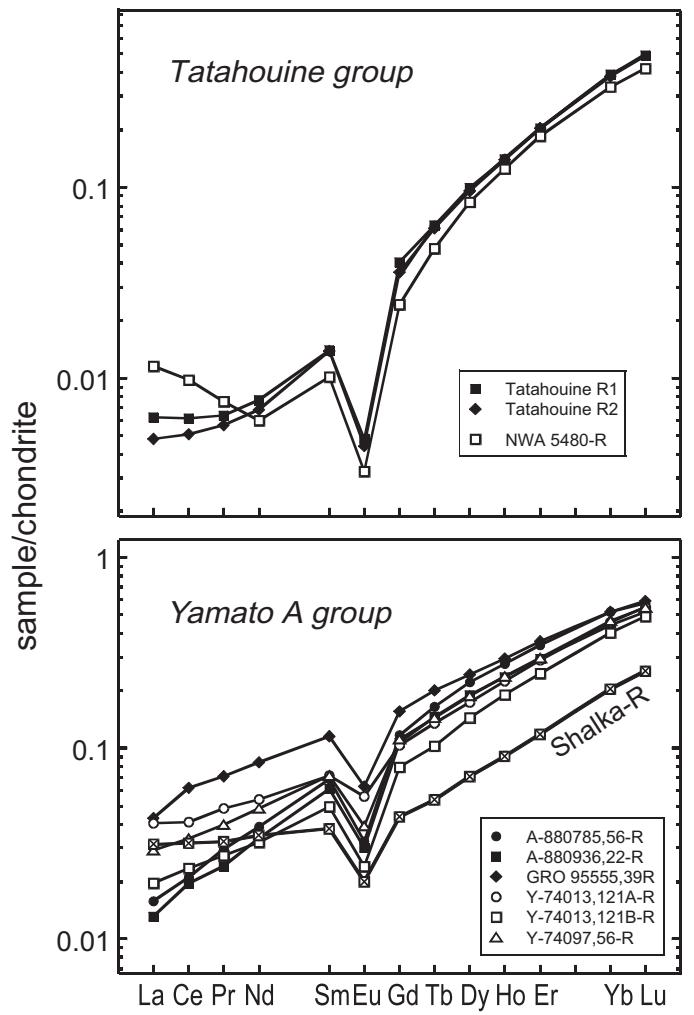

Fig. 4. REE patterns of the Tatahouine group and Yamato-A group diogenites (residues after leaching). The REE pattern of Shalka is shown for comparison. The reference chondrite is from Evensen et al. (1978). The $2 \sigma$ errors for the new analyses are equivalent to the size of the data points.

display REE patterns with very deep Eu anomalies $\left(\mathrm{Eu} / \mathrm{Eu}^{*}=0.08-0.16\right)$, and marked light REE depletions $\left((\mathrm{La} / \mathrm{Sm})_{n}=0.08-0.23\right)$ Fig. 6.

\subsubsection{NWA 5613 group}

The orthopyroxenes of the diogenites from this group (NWA 3143, NWA 4272, NWA 5613, A-881838, A881839, A-881944, MIL 07003) display the same kind of REE patterns as those of the Johnstown group with very low $\mathrm{Eu} / \mathrm{Eu}^{*}$ values $\left(\mathrm{Eu} / \mathrm{Eu}^{*}=0.04-0.12\right)$ but with less pronounced light REE depletions $\left((\mathrm{La} / \mathrm{Sm})_{n}=0.26-0.36\right)$.

The REE patterns of the few others samples analyzed during the course of the study cannot be classified in the previous groups. The shapes of the REE patterns of A-87147, A-88377 and NWA 4664 are like those of the NWA 5613 group but the negative Eu anomalies are much less pronounced (Fig. 7). ALHA 77256 (Fig. 7) and Shalka (Fig. 4) cannot be grouped easily with other samples. Notice that Shalka displays the same heavy REE enrichment as the Yamato-A group pyroxenes but is not light REE depleted (Fig. 4). Finally, MET 00424 is by far the most unusual sample (Fig. 8). This diogenite displays extremely low REE abundances and the most fractionated heavy REE abundances. Unfortunately, the light REE concentrations were too low to be correctly determined with previous procedures. We have separated and concentrated the REEs 


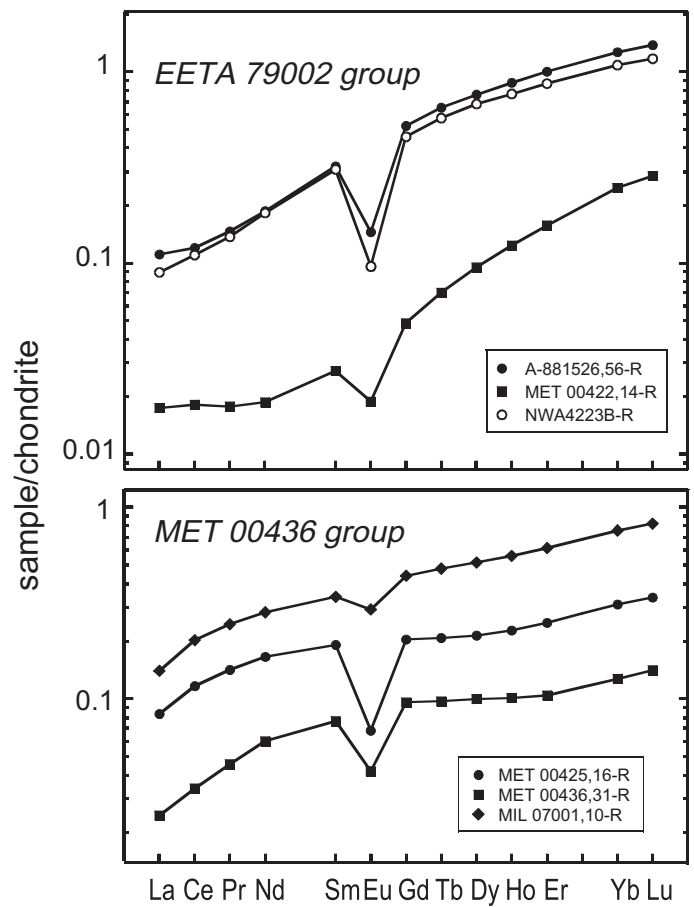

Fig. 5. REE patterns of the EETA79002 group and MET 00436 group diogenites (residues after leaching). The reference chondrite is from Evensen et al. (1978). The $2 \sigma$ errors for the new analyses are equivalent to the size of the data points.

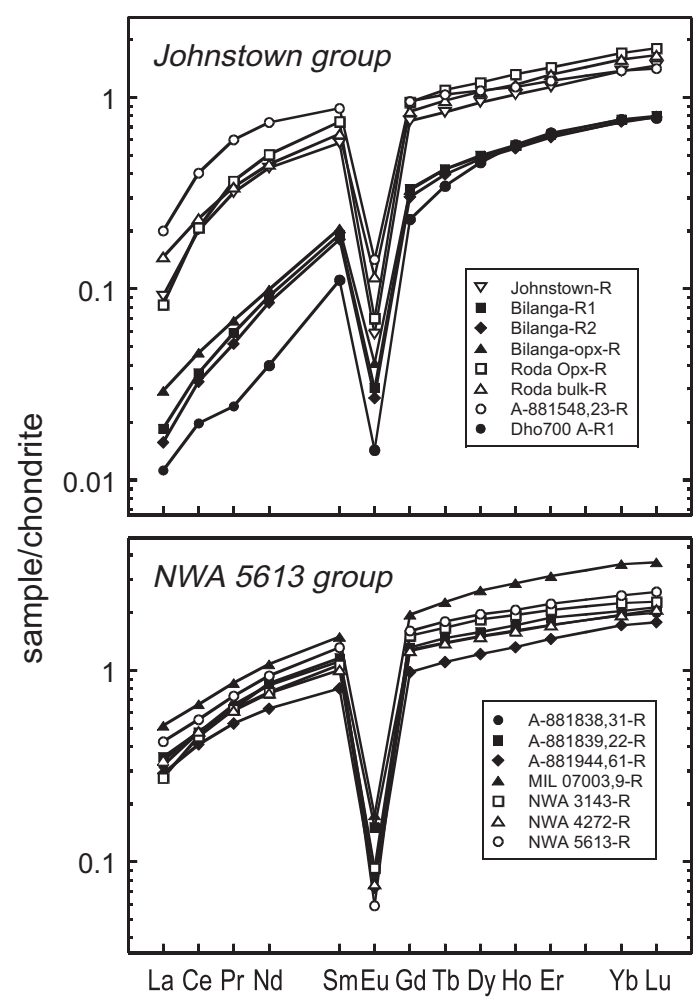

Fig. 6. REE patterns of the Johnstown group and NWA 5613 group diogenites (residues after leaching). The reference chondrite is from Evensen et al. (1978). The $2 \sigma$ errors for the new analyses are equivalent to the size of the data points.

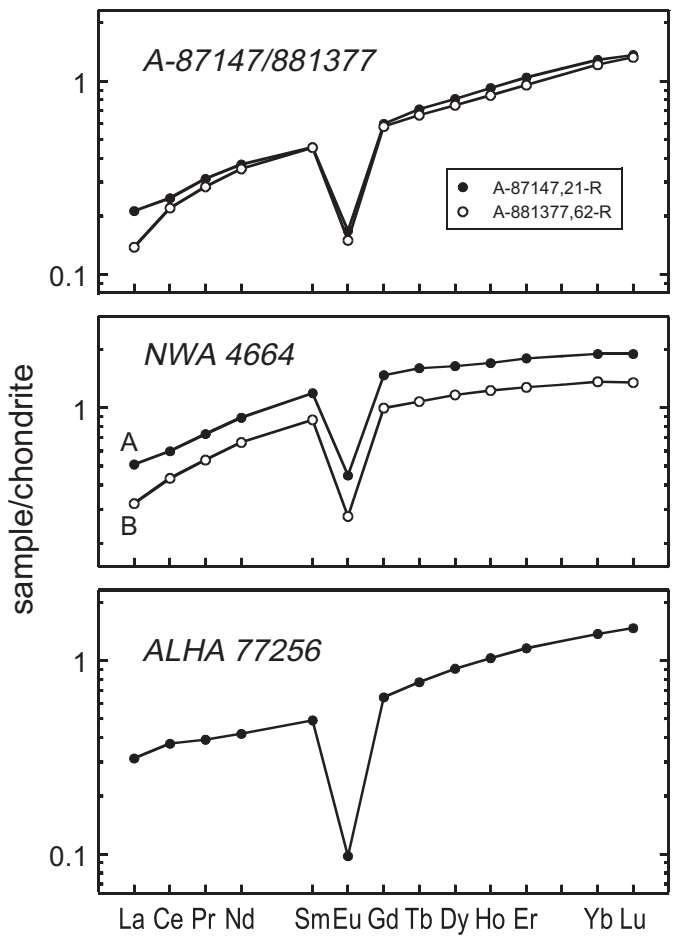

Fig. 7. REE patterns of the ALHA 77256, NWA 4664, A-87147 and A-881377 diogenites (residues after leaching). The reference chondrite is from Evensen et al. (1978). The $2 \sigma$ errors for the new analyses are equivalent to the size of the data points.

from the solutions prepared with the unleached powder and from the residue after leaching, and we have determined the abundances of all the REEs. These new data confirm the results obtained previously by Mittlefehldt (2002) and Barrat et al. (2008) (Fig. 8). Both fractions display the same kind of pattern, i.e., with a strong heavy REE enrichment $\left((\mathrm{Gd} / \mathrm{Lu})_{n}=0.004-0.008\right)$, very low middle REE abundances $\left(<10^{-3} \times \mathrm{CI}\right)$ and a marked light REE enrichment $\left((\mathrm{La} / \mathrm{Sm})_{n}=3.5-4.3\right)$. Although the $\mathrm{Eu}$ abundances we have obtained are just indicative, it is certain that the two analyzed fractions do not exhibit deep Eu anomalies. The unleached fraction displays the same heavy REE abundances as the residue, but about twice more light REEs. The difference could be explained by the effect of traces of terrestrial secondary phases (rust) in the unleached powder. Moreover, these REE patterns cannot be explained by any simple model. One may speculate that such U- or V-shape patterns can result of interactions between a strongly heavy REE enriched component (i.e., a pure orthopyroxenite) with a light REE enriched component (i.e., an infiltrated melt?), but this hypothesis cannot be discussed with this single sample. It has been previously suggested that MET 00424 is paired with MET 00436 (Warren et al., 2009), but REE abundances rule out this possibility.

\subsection{Origin of the Eu anomalies}

Several mechanisms may be considered to be responsible for the observed range of $\mathrm{Eu} / \mathrm{Eu}^{*}$ values, and are discussed below. 


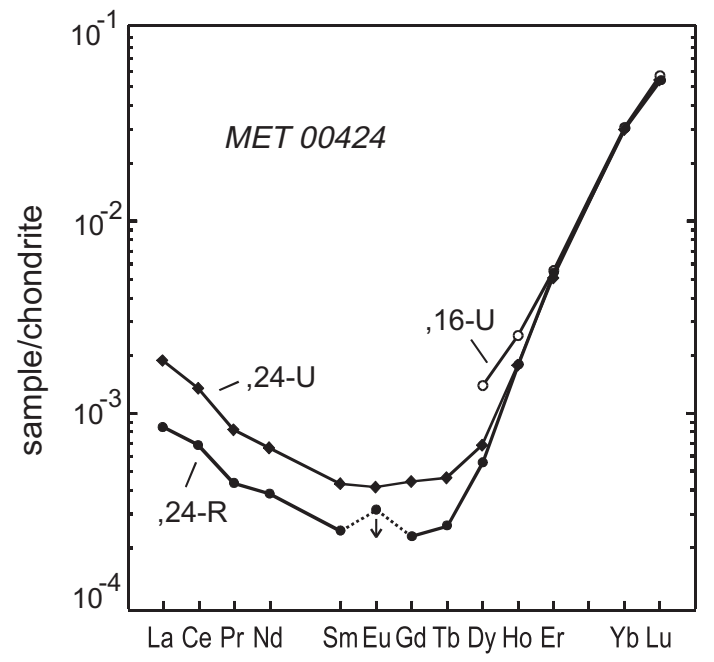

Fig. 8. REE patterns of the MET 00424 diogenite (R, residue after leaching; $\mathrm{U}$, unleached powder). The analysis obtained previously on an unleached sample $(, 16)$ is shown for comparison (Barrat et al., 2008). The reference chondrite is from Evensen et al. (1978).

Firstly, diogenites contain occasionally sparse plagioclase grains, and this phase displays large positive $\mathrm{Eu}$ anomalies (Mittlefehldt, 1994). Variable amounts of plagioclase in the analyzed fractions can potentially be the cause of the range of $\mathrm{Eu} / \mathrm{Eu}^{*}$ values measured here: calculations show that only $2 \mathrm{wt} \%$ of plagioclase are able to compensate the deep negative Eu anomalies displayed by the orthopyroxenes in Dhofar 700 (Fig. 9). Although traces of plagioclase could explain the limited spread of values obtained for some plagioclase-bearing diogenites (the Yamato-type A ones for example), this explanation cannot account for the full range of $\mathrm{Eu} / \mathrm{Eu}^{*}$ values. Most of the analyzed fractions, some displaying among the lowest $\mathrm{Eu} / \mathrm{Eu}^{*}$ values (e.g., NWA 5613, orthopyroxene fractions from Johnstown or Roda) and others displaying the highest ones (e.g., Shalka, MIL 07001, NWA 1461), were totally devoid of plagioclase.

The immediate question that must be addressed, is whether or not the orthopyroxenes in diogenites preserve some of the pristine magmatic features of their trace element abundances. The involvement of a small amount of trapped melt and subsolidus reequilibration between orthopyroxene and the interstitial phases can modify the concentrations of incompatible elements of the orthopyroxenes severely during the cooling of diogenites (Treiman, 1996; Barrat, 2004). The $\mathrm{Eu} / \mathrm{Eu}^{*}$ ratios are sensitive to these processes. In the absence of plagioclase, the involvement of a few percent of trapped melt can increase the $\mathrm{Eu} / \mathrm{Eu}^{*}$ ratio of the orthopyroxene, and could explain some of our results (MIL 07001, NWA 1461). If plagioclase is an interstitial phase, the opposite effect is expected. However, this effect cannot account for the lowest $\mathrm{Eu} / \mathrm{Eu}^{*}$ values shown by the diogenitic orthopyroxenes because plagioclase is at best a minor phase in all the diogenites we have analyzed, and is not abundant enough to have a significant effect on the $\mathrm{Eu} / \mathrm{Eu}^{*}$ values of the orthopyroxenes. Moreover, the orthopyroxenes in Dhofar 700, an unbrecciated diogenite

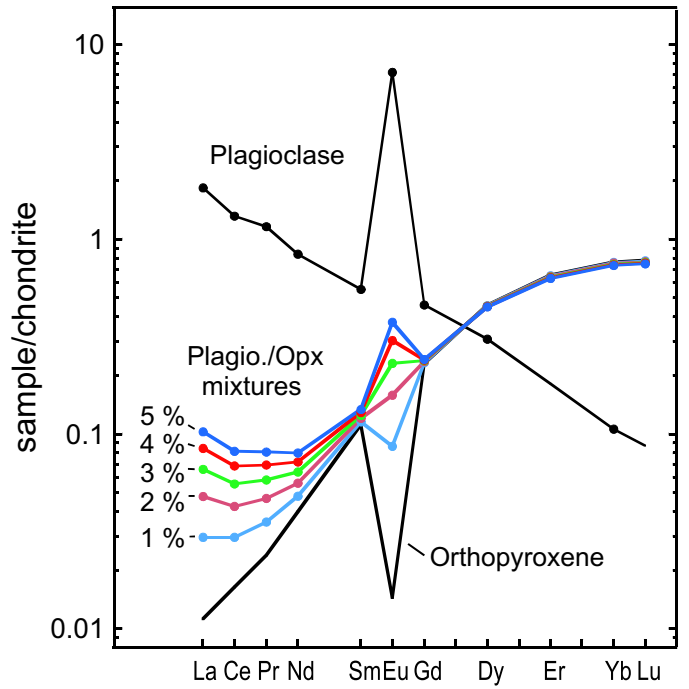

Fig. 9. The effects of traces of plagioclase in a diogenite split. The calculations were made using orthopyroxene (Ce was interpolated between La and Pr because of terrestrial weathering) and plagioclase from Dhofar 700. The deep negative Eu anomaly of the pyroxene is totally masked with only $2 \%$ of plagioclase. The reference chondrite is from Evensen et al. (1978).

containing minor interstitial plagioclase, and whose $\mathrm{Eu} /$ $\mathrm{Eu}^{*}$ ratios $(=0.09-0.13)$ are among the lowest measured, still display pristine zoning for major elements. Thus, the very low negative $\mathrm{Eu}$ anomalies shown by the diogenitic orthopyroxenes are not generated by subsolidus processes, and are more likely a pristine feature.

The behavior of Eu during the crystallization of low-Ca pyroxene is strongly dependant on the $f \mathrm{O}_{2}$, and is decoupled from Sm and $\mathrm{Gd}$, its neighboring REEs, in reducing conditions (Schwandt and McKay, 1998; McCanta et al., 2004). Thus, the wide range of Eu anomalies displayed by the diogenitic orthopyroxenes could be explained by a diversity of redox states during the crystallization of the diogenitic systems. However, the striking correlation between the $\mathrm{Sr} / \mathrm{Eu}^{*}$ ratios, which are insensitive to the redox state (McKay et al., 1994), and the $\mathrm{Eu} / \mathrm{Eu}^{*}$ ratios, rules out this possibility (Fig. 10).

Furthermore, the correlation between the $\mathrm{Sr} / \mathrm{Eu}^{*}$ and $\mathrm{Eu} / \mathrm{Eu}^{*}$ ratios could be the fingerprint of the involvement of plagioclase during the genesis of the parental melts of diogenites. Two scenarios can be proposed. Firstly, low $\mathrm{Eu} / \mathrm{Eu}^{*}$ and $\mathrm{Sr} / \mathrm{Eu}^{*}$ ratios in a melt can be achieved by the removal of large amounts of plagioclase. Secondly, melts displaying low $\mathrm{Sr} / \mathrm{Eu}^{*}$ and $\mathrm{Eu} / \mathrm{Eu}^{*}$ ratios can be produced by partial melting of a plagioclase-bearing ultramafic source while plagioclase is a residual phase. In both cases, these processes are inappropriate here. Plagioclase is clearly not an early crystallizing phase in the diogenitic melts (e.g., Mittlefehldt, 1994; Bowman et al., 1999; Beck and McSween, 2010). Consequently, the parental melts of the diogenites were certainly not in equilibrium with a plagioclase-bearing source. For the same reason, the crystallization of large amounts of plagioclase before orthopyroxene from these melts is just not possible. 


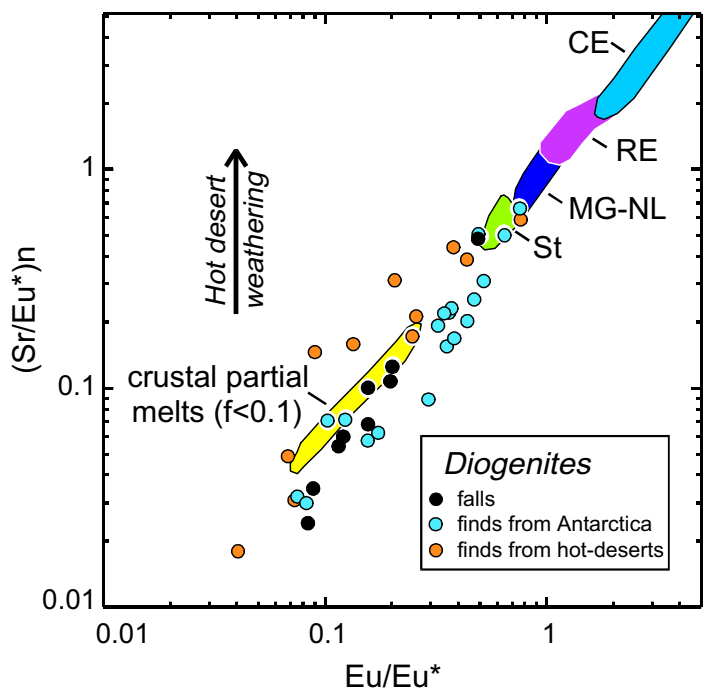

Fig. 10. Sr/Eu* (chondrite normalized) vs. $\mathrm{Eu} / \mathrm{Eu}^{*}$ plot for diogenites (residues after leaching, this study), and eucrites (MGNL, Main Group-Nuevo Laredo; St, Stannern trend eucrites; RE, residual eucrites; CE, cumulate eucrites; Shimizu and Masuda, 1986; Barrat et al., 2000, 2003, 2007; Yamaguchi et al., 2009). The $2 \sigma$ errors are equivalent to the size of the data points.

Diogenites crystallized probably at the same $f \mathrm{O}_{2}$ conditions as eucrites, close to the iron-wüstite buffer down to 1 log-unit below it (Stolper, 1977; Hewins and Ulmer, 1984). Under these conditions, a melt with no Eu anomaly is in equilibrium with a low-Ca pyroxene with a $\mathrm{Eu} / \mathrm{Eu}^{*}$ ratio close to 0.2-0.4 (Schwandt and McKay, 1998; McCanta et al., 2004). Therefore, the very large negative Eu anomalies exhibited by some diogenitic orthopyroxenes suggest that they formed from melts displaying extremely low Eu/ Eu* ratios, as low as $0.1-0.2$. How can the parental melts of some diogenites achieve such deep negative Eu anomalies without the direct involvement of plagioclase? The only plausible solution to this question is that these melts have been contaminated by component(s) displaying an extremely large negative Eu anomaly. Such components could have been easily generated on Vesta (Barrat et al., 2007; Yamaguchi et al., 2009). Melts displaying very high REE abundances, very low $\mathrm{Eu} / \mathrm{Eu}^{*}$ and low $\mathrm{Sr} / \mathrm{Eu}^{*}$ ratios, have probably been produced by low degrees of melting of the eucritic crust (Figs. 10 and 11). The intrusion of hot diogenitic parental melts into the eucritic crust could have increased the temperatures of the country rocks above their solidus, resulting in a localized partial melting of the crust.

The modeling of the contamination of the parental melts of diogenites by a crustal partial melt is not a trivial task. Firstly, the trace element abundances of diogenites indicate a diversity of their parental melts, whose compositions are still a matter of debate. If our hypothesis is correct, the uncontaminated or the least contaminated parental melts of diogenites should display among the lowest trace element abundances. For the calculation purposes, we have chosen the parental melts of Tatahouine and Y-74097 whose REE abundances are low. We estimate their trace element abundances from the partition coefficients obtained by Schwandt and McKay (1998). Notice that parental melts of diogenites with much lower REE abundances have been generated (e.g., the melt in equilibrium with MET 00424). Secondly, the eucritic crust is certainly heterogeneous and is made of a variety of flows and intrusions (from $\mathrm{Mg}$-rich cumulate eucrite to Nuevo Laredo and Stannern trend eucrites), displaying a wide range of REE and other trace element abundances. Thus, the melts possibly generated during the

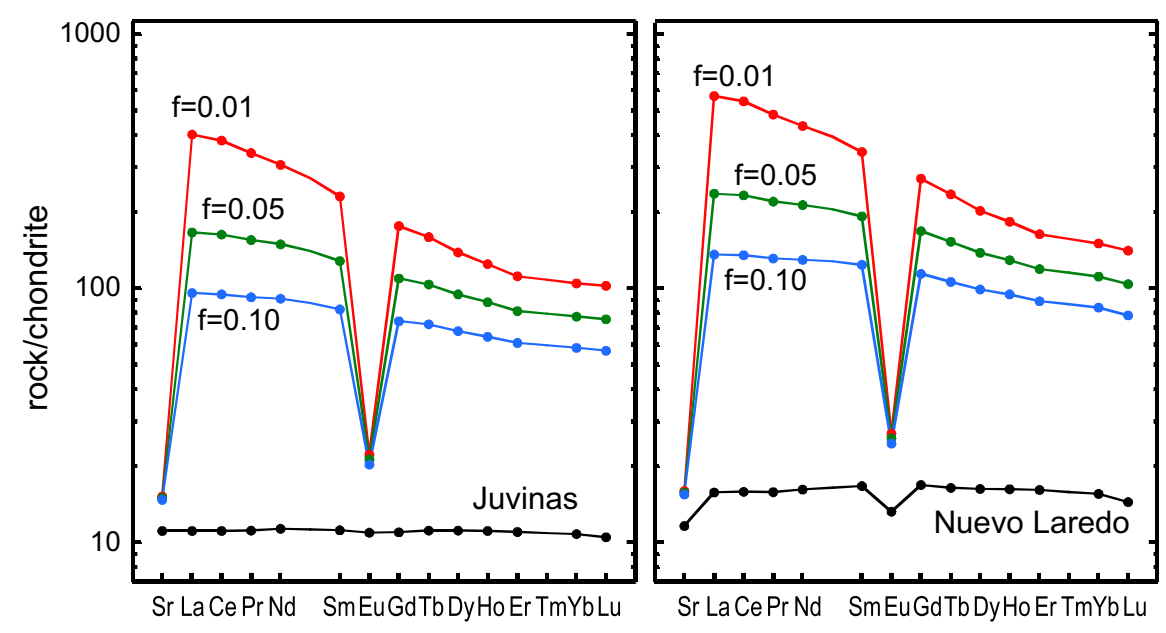

Fig. 11. REE patterns of calculated magmas derived by modal partial melting of two typical noncumulate eucrites. The reference chondrite is from Evensen et al. (1978). Because the compositions of melts generated by the melting of a eucrite have not yet been determined experimentally, we estimated their trace element abundances theoretically using the same model and parameters as Barrat et al. (2007). We used the compositions of Juvinas and Nuevo Laredo, and estimated the compositions of the melts after 1\%, $5 \%$ and $10 \%$ of partial melting. In reality, the melting of a eucrite would be more complex than is considered by this model. The involvement of REE-rich accessory phases such as phosphates would certainly be significant at very low degrees of melting, and melts with higher REE abundances but similar Eu and Sr anomalies are expected. However, these calculations should give a realistic picture of the REE abundances of these partial melts, and demonstrate that they are characterized by very high REE abundances and huge negative Eu and $\mathrm{Sr}$ anomalies. 

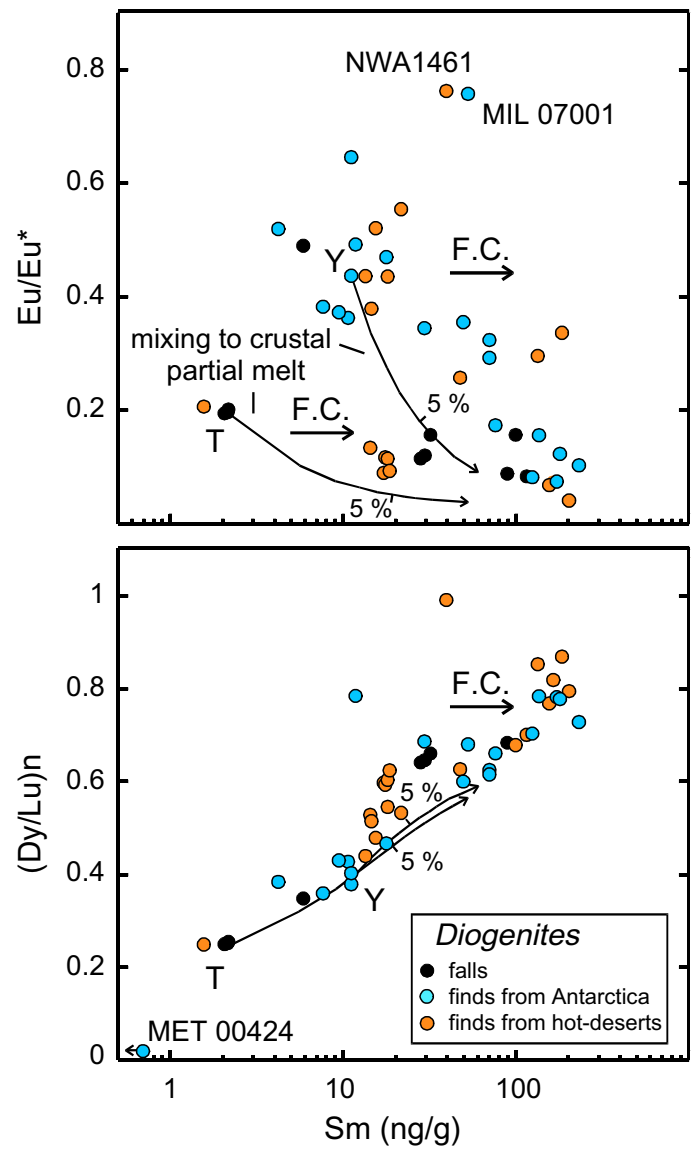

Fig. 12. $\mathrm{Eu} / \mathrm{Eu}^{*}$ and $(\mathrm{Dy} / \mathrm{Lu})_{n}$ vs. Sm plot for diogenites. The mixing curves correspond to the composition of the orthopyroxenes in equilibrium respectively with the parental melts of the Tatahouine (T) and Yamato-74097 (Y) diogenites contaminated with a crustal partial melt. The parental melts of the diogenites were estimated from the REE abundances of the residues and orthopyroxene partition coefficients (Schwandt and McKay, 1998). A magma obtained after $2 \%$ of melting of the Juvinas eucrite has been chosen as contaminant. In addition to the contamination of the parental magmas of diogenites by crustal partial melts, fractional crystallization explains part of the spread of data. The $2 \sigma$ errors are equivalent to the size of the data points.

melting of the eucritic crust displayed certainly a wide range of REE abundances, as exemplified in Fig. 10. We have used the same parameters as Barrat et al. (2007) for the melting model (Barrat et al., 2007, pp. 4118-4119), and a magma obtained after $2 \%$ of partial melting of the Juvinas eucrite has been used for the calculations. However, because phosphates are the main carriers of REE in eucrites (Delaney et al., 1984; Hsu and Crozaz, 1996), and are among the first phases involved during the partial melting (Yamaguchi and Mikouchi, 2005), magmas generated by very low degree of partial melting (less than $5 \%$ ) have probably much higher light REE abundances and possibly even lower negative $\mathrm{Eu}$ anomalies than the compositions estimated from simple partial melting calculations. Simple mixing calculations indicate that it is possible to produce orthopyroxenes with very low $\mathrm{Eu} / \mathrm{Eu}^{*}$ ratios from a diogenitic parental melt if the latter has assimilated less than $10 \%$ of such crustal partial melts (Fig. 12). Despite the uncertainties due to the lack of knowledge of the parental melts of the diogenites, the uncertainties about the composition of the assimilated melts, and the partition coefficients, the low levels of contamination suggested by the calculations indicate that it is a viable mechanism for producing the range of the $\mathrm{Eu} / \mathrm{Eu}^{*}$ values of the diogenitic orthopyroxenes. More complex models of contamination processes, such as combination of assimilation of a contaminant by the melt and fractional crystallization (e.g., DePaolo, 1981) do not alter this conclusion.

Furthermore, diogenites display an exceptional range of heavy REE enrichments with $(\mathrm{Dy} / \mathrm{Lu})_{n}$ ratios from values close to $0.02-1$. Interestingly, the $(\mathrm{Dy} / \mathrm{Lu})_{n}$ ratios are correlated with the Sm abundances (Fig. 12) and such correlation could be at least partly explained by the contamination of the parental melts of diogenites by the crustal partial melts. Our calculations suggest that the contamination hypothesis can satisfactorily explain a significant part of the trend but fails to account for the whole range of the values. We suspect that the (Dy/Lu) ratios of the crustal partial melts are not satisfactorily estimated by the model (phosphate effect for low degrees of melting?). Experimental work is needed to estimate more precisely the compositions of the melts formed during the melting of a eucrite.

\section{CONCLUSIONS}

The very low $\mathrm{Eu} / \mathrm{Eu}^{*}$ values displayed by some diogenites and their orthopyroxenes are best explained by the contamination of their parental magmas by melts derived from the eucritic crust. This conclusion has profound implications for our understanding of the magmatic history and the structure of the crust of Vesta. Firstly, geochronological studies have demonstrated that diogenites are ancient rocks, but the uncertainties on the crystallization ages do not allow a relative chronology with the eucrites (Lugmair and Shukolyukov, 1998). Although diogenites cannot be dated precisely, our results imply that they formed contemporaneously to, or more likely, after eucrites. Secondly, it is generally believed that Vesta displays a well stratified structure, with the upper part composed, from the top to the bottom, of a variety of eucrites, progressively more metamorphosed with depth, followed by diogenites which are seen as the deepest samples yet available of the body (Takeda, 1979). This view has been strengthened by models of crystallization of a global magma ocean (Righter and Drake, 1997; Ruzicka et al., 1997) which satisfactorily explain the short duration of eucritic magmatism (e.g., Lugmair and Shukolyuokov, 1998; Bizzaro et al., 2005; Misawa et al., 2005), the low siderophile trace element abundances of the eucrites (Righter and Drake, 1997), and the homogeneity of the $\Delta^{17} \mathrm{O}$ values of the HED lithologies (Greenwood et al., 2005; Scott et al., 2009). A global diogenitic layer localized below the eucritic crust is assumed by these models (Fig. 13A). Although the vast majority of the known diogenites certainly formed at depth, a few samples (e.g., Garland (Fowler et al., 1994), Dho 700, NWA 4215) display zoned pyroxenes which indicate that they 

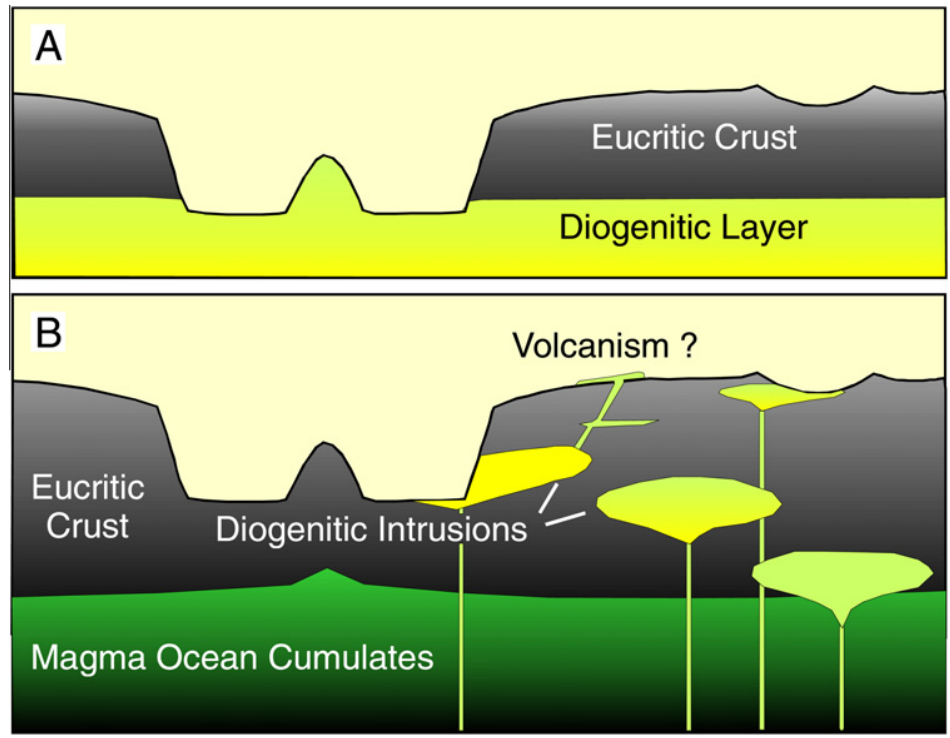

Fig. 13. Models of the crust of Vesta. Two hypothetical impact craters are shown: a large one with a central peak, and a smaller one. The vertical and horizontal scales are arbitrary. It is often assumed that the crust of Vesta is layered (e.g., Takeda, 1979). Following this view, the upper part is composed of various types of eucrites and covers a thick layer of diogenites which is exposed on the floor of the deepest impact craters (A). Our data demonstrate that the parental melts of diogenites have been contaminated by crustal partial melts and such a contamination implies that they have intruded the eucritic crust (B). Thus, exposures of diogenites are probably not limited to the largest impact craters, but could be found in less deep craters. Lavas associated with the diogenitic magmatism could have been locally erupted.

cooled much faster than other diogenites, and formed more likely within shallow intrusions (Barrat et al., 2006). Therefore, these petrological considerations and the chemical constraints presented here show that the parental melts of diogenites have intruded the eucritic crust, pointing to a different picture of the structure of the Vestan crust (Fig. 13B). At present, the available Hubble Space Telescope images have clearly demonstrated that the surface of Vesta is highly impacted. The largest impact craters could have exposed deep crustal areas, especially in the southern hemisphere where a huge impact basin, $460 \mathrm{~km}$ in diameter, has been described (Thomas et al., 1997). The available geological models deduced from these images are too uncertain to constrain the structure of the crust (Binzel et al., 1997; Li et al., 2010). The planned remote sensing studies that will be undertaken next year by the Dawn spacecraft are necessary to describe the various crustal units exposed by the craters (Russell et al., 2007). The geochemistry of HED meteorites thus may provide a framework to better understand the observations of the Dawn mission.

\section{ACKNOWLEDGMENTS}

Financial support from the Programme National de Planétologie de l'Institut National des Sciences de l'Univers is acknowledged. We thank the National Institut of Polar Research, the Meteorite Working Group (NASA), the Muséum national d'Histoire Naturelle de Paris, the American Museum of Natural History, New York, the Royal Ontario Museum, Alain Carion, Michel Franco, and Ali Hmani for providing the meteorites samples; R. Hewins, A.W. Beck, M. Toplis, R.C. Greenwood for critically reading an early version of the manuscript. We thank C. Koeberl for the editorial handling, C. Floss and K. Righter for their critical and con- structive reviews. J.A.B. acknowledges Pascale Barrat for her help. This research has made use of NASA's Astrophysics Data System Abstract Service.

\section{REFERENCES}

Barrat J. A. (2004) Determination of the parental magmas of HED cumulates: the effects of interstitial melts. Meteorit. Planet. Sci. 39, 1767-1779.

Barrat J. A. and Bollinger C. (2010) Geochemistry of the martian meteorite ALH84001, revisited. Meteorit. Planet. Sci., doi:10.1111/j.1945-5100.2010.01042.x.

Barrat J. A., Keller F., Amossé J., Taylor R. N., Nesbitt R. W. and Hirata T. (1996) Determination of rare earth elements in sixteen silicate reference samples by ICP-MS using a Tm addition and an ion exchange chromatography procedure. Geostand. Newsl. 20, 133-139.

Barrat J. A., Gillet Ph., Lesourd M., Blichert-Toft J. and Poupeau G. R. (1999) The Tatahouine diogenite: mineralogical and chemical effects of sixty-three years of terrestrial residence. Meteorit. Planet. Sci. 34, 91-97.

Barrat J. A., Blichert-Toft J., Gillet Ph. and Keller F. (2000) The differentiation of eucrites: the role of in-situ crystallization. Meteor. Planet. Sci. 35, 1087-1100.

Barrat J. A., Jambon A., Bohn M., Blichert-Toft J., Sautter V., Göpel C., Gillet Ph., Boudouma O. and Keller F. (2003) Petrology and geochemistry of the unbrecciated achondrite North West Africa 1240 (NWA 1240): an HED parent body impact melt. Geochim. Cosmochim. Acta 67, 3959-3970.

Barrat J. A., Beck P., Bohn M., Cotten J., Gillet Ph., Greenwood R. C. and Franchi I. A. (2006) Petrology and geochemistry of the fine-grained, unbrecciated diogenite Northwest Africa 4215. Meteorit. Planet. Sci. 41, 1045-1057.

Barrat J. A., Yamaguchi A., Greenwood R. C., Bohn M., Cotten J., Benoit M. and Franchi I. A. (2007) The Stannern trend 
eucrites: contamination of main group eucritic magmas by crustal partial melts. Geochim. Cosmochim. Acta 71, 4108-4124.

Barrat J. A., Yamaguchi A., Benoit M., Cotten J. and Bohn M. (2008) Geochemistry of diogenites: still more diversity in their parental melts. Meteorit. Planet. Sci. 43, 1759-1775.

Barrat J. A., Yamaguchi A., Greenwood R. C., Bollinger C., Bohn M. and Franchi I. A. (2009) Trace element geochemistry of Krich impact spherules from howardites. Geochim. Cosmochim. Acta 73, 5944-5958.

Beck A. W. and McSween, Jr., H. Y. (2010) Diogenites as polymict breccias composed of orthopyroxenite and harzburgite. Meteorit. Planet. Sci., doi:10.1111/j.1945-5100.2010.01061.x.

Binzel R. P., Gaffey M. J., Thomas P. C., Zellner B. H., Storrs A. D. and Wells E. N. (1997) Geological mapping of Vesta from 1994 Hubble Space Telescope images. Icarus 128, 95-103.

Bizzaro M., Baker J. A., Haack H. and Lundgaard L. (2005) Rapid timescales for accretion and melting of differentiated planetesimals inferred from $26 \mathrm{Al}-26 \mathrm{Mg}$ chronometry. Astrophys. $J$. 632, L41-L44.

Bowman L. E., Papike J. J. and Spilde M. N. (1999) Diogenites as asteroidal cumulates: insights from spinel chemistry. $\mathrm{Am}$. Mineral. 84, 1020-1026.

Delaney J. S., Prinz M. and Takeda H. (1984) The polymict eucrites. In Proceedings of the 15th Lunar Planetary Science Conference, J. Geophys. Res., Suppl. 89, pp. C251-C288.

DePaolo D. J. (1981) Trace element and isotopic effects of combined wall rock assimilation and fractional crystallization. Earth Planet. Sci. Lett. 53, 189-202.

Domanik K., Kolar S., Musselwhite D. and Drake M. J. (2004) Accessory silicate mineral assemblages in the Bilanga diogenite: a petrographic study. Meteorit. Planet. Sci. 39, 567-579.

Drake M. J. (2001) The eucrite/Vesta story. Meteorit. Planet. Sci. 36, 501-513.

Evensen N. M., Hamilton P. J. and O'Nions R. K. (1978) Rare earth abundances in chondritic meteorites. Geochim. Cosmochim. Acta 42, 1199-1212.

Floran R. J., Prinz M., Hlava P. F., Keil K., Spettel B. and Wänke H. (1981) Mineralogy, petrology, and trace element geochemistry of the Johnstown meteorite: a brecciated orthopyroxenite with siderophile and REE-rich components. Geochim. Cosmochim. Acta 45, 2385-2391.

Fowler G. W., Papike J. J., Shearer C. K. and Spilde M. N. (1994) Diogenites as asteroidal cumulates. Insights from orthopyroxene major and minor element chemistry. Geochim. Cosmochim. Acta 58, 3921-3929.

Fowler G. W., Papike J. J., Spilde M. N. and Shearer C. K. (1995) Diogenites as asteroidal cumulates. Insights from orthopyroxene trace element chemistry. Geochim. Cosmochim. Acta 59, 3071-3084.

Fukuoka T., Boynton W. V., Ma M. S. and Schmitt R. A. (1977) Genesis of howardites, diogenites and eucrites. In Proceedings of the Eighth Lunar Science Conference, pp. 187-210.

Greenwood R. C., Franchi I. A., Jambon A. and Buchanan P. C. (2005) Widespread magma oceans on asteroidal bodies in the early solar system. Nature $\mathbf{4 3 5}, 916-918$.

Hewins R. H. and Ulmer G. C. (1984) Intrinsic oxygen fugacities of diogenites and mesosiderite clasts. Geochim. Cosmochim. Acta 48, $1555-1560$.

Hsu W. and Crozaz G. (1996) Petrogenesis of eucrites: I. Noncumulate eucrites. Geochim. Cosmochim. Acta 60, 4571-4591.

Li J. Y., McFadden L. A., Thomas P. C., Mutchler M. J., Parker J. W., Young E. F., Russell C. T., Sykes M. V. and Schmidt B. E. (2010) Photometric mapping of asteroid (4) Vesta's southern hemisphere with Hubble Space Telescope. Icarus. doi:10.1016/ j.icarus.2010.02.008.
Lugmair G. W. and Shukolyukov A. (1998) Early solar system timescales according to ${ }^{53} \mathrm{Mn}-{ }^{53} \mathrm{Cr}$ systematics. Geochim. Cosmochim. Acta 62, 2863-2886.

McCanta M. C., Rutherford M. J. and Jones J. H. (2004) An experimental study of rare earth element partitioning between a shergottite melt and pigeonite: implications for the oxygen fugacity of the martian interior. Geochim. Cosmochim. Acta $\mathbf{6 8}$, 1943-1952.

McKay G. A., Le L., Wagstaff J. and Crozaz G. (1994) Experimental partitioning of rare earth elements and strontium: constraints on petrogenesis and redox conditions during crystallization of Antarctic angrite Lewis Cliff 86010. Geochim. Cosmochim. Acta 58, 2911-2919.

McSween, Jr., H. Y., Mittlefehldt D. W., Beck A. W., Mayne R. G., McCoy T. J. (2010) HED meteorites and their relationship to the geology of Vesta. Space Sci. Rev. doi:10.1007/s11214010-9637-z.

Misawa K., Yamaguchi A. and Kaiden H. (2005) U-Pb and ${ }^{207} \mathrm{~Pb}-{ }^{206} \mathrm{~Pb}$ ages of zircons from basaltic eucrites: implications for early basaltic volcanism on the eucrite parent body. Geochim. Cosmochim. Acta 69, 5847-5861.

Mittlefehldt D. W. (1994) The genesis of diogenites and HED parent body petrogenesis. Geochim. Cosmochim. Acta 58, 15371552.

Mittlefehldt D. W. (2000) Petrology and geochemistry of the Elephant Moraine A79002 diogenite: a genomict breccia containing a magnesian harzburgite component. Meteorit. Planet. Sci. 37, 345-369.

Mittlefehldt D.W. (2002) Geochemistry of new, unusual diogenites and constraints on diogenite genesis (abstract). 65th Annual Meeting of the Meteoritical Society. Meteorit. Planet. Sci. 37, A100.

Righter K. and Drake M. J. (1997) A magma ocean on Vesta: core formation and petrogenesis of eucrites and diogenites. Meteorit. Planet. Sci. 32, 929-944.

Russell C. T. et al. (2007) Dawn mission to Vesta and Ceres. Earth Moon Planets 101, 65. doi:10.1007/s11038-007-9151-9.

Ruzicka A., Snyder G. A. and Taylor L. A. (1997) Vesta as the howardite, eucrite and diogenite parent body: implications for the size of a core and for large-scale differentiation. Meteorit. Planet. Sci. 32, 825-840.

Sack R. O., Azeredo W. J. and Lipschutz M. E. (1991) Olivine diogenites: the mantle of the eucrite parent body. Geochim. Cosmochim. Acta 55, 1111-1120.

Schwandt C. S. and McKay G. A. (1998) Rare Earth element partition coefficients from enstatite/melt synthesis experiments. Geochim. Cosmochim. Acta 62, 2845-2848.

Scott E. R. D., Greenwood R. C., Franchi I. A. and Sanders I. S. (2009) Oxygen isotopic constraints on the origin and parent bodies of eucrites, diogenites, and howardites. Geochim. Cosmochim. Acta 73, 5835-5853.

Shearer C. K., Burger P. and Papike J. J. (2010) Petrogenetic relationships between diogenites and olivine diogenites: implications for magmatism on the HED parent body. Geochim. Cosmochim. Acta 74, 4865-4880.

Shimizu H. and Masuda A. (1986) REE patterns of eucrites and their genetic implications. Geochim. Cosmochim. Acta 50, 24532460.

Stolper E. (1977) Experimental petrology of eucrite meteorites. Geochim. Cosmochim. Acta 41, 587-611.

Takeda H. (1979) A layered-crust model of a howardite parent body. Icarus 40, 455-470.

Takeda H., Mori H. and Yanai K. (1981) Mineralogy of the Yamato diogenites as possible pieces of a single stone. Mem. Natl. Inst. Polar Res. 20, 81-99 (special issue). 
Thomas P. C., Binzel R. P., Gaffey M. J., Storrs A. D., Wells E. N. and Zellner B. H. (1997) Impact excavation on asteroid 4-Vesta: Hubble Space Telescope results. Science 277, 1492-1495.

Treiman A. H. (1996) The perils of partition: difficulties in retrieving magma compositions from chemically equilibrated basaltic meteorites. Geochim. Cosmochim. Acta 60, 147-155.

Warren P. H. (1997) Magnesium oxide-iron oxide mass balance constraints and a more detailed model for the relationship between eucrites and diogenites. Meteorit. Planet. Sci. 32, 945963.

Warren P. H., Kallemeyn G. W., Huber H., Ulff-Møller F. and Choe W. (2009) Siderophile and other geochemical constraints on mixing relationships among HED-meteoritic breccias. Geochim. Cosmochim. Acta 73, 5918-5943.
Yamaguchi A. and Mikouchi T. (2005) Heating experiments of the $\mathrm{HaH} 262$ eucrite and implication for the metamorphic history of highly metamorphosed eucrites. Lunar Planet. Sci. 36, CD\#1574 (abstr.).

Yamaguchi A., Barrat J. A., Greenwood R. C., Shirai N., Okamoto C., Setoyanagi T., Ebihara M., Franchi I. A. and Bohn M. (2009) Crustal partial melting on Vesta: evidence from highly metamorphosed eucrites. Geochim. Cosmochim. Acta 73, 7162-7182.

Associate editor: Christian Koeberl 